\title{
Mathematical Modeling on Rotational Magneto-Thermoelastic Phenomenon under Gravity and Laser Pulse considering Four Theories
}

\author{
Hammad Alotaibi, ${ }^{1}$ S. M. Abo-Dahab ${ }^{10,3}$ E. M. Khalil, ${ }^{1}$ S. Abdel-Khalek, ${ }^{1}$ \\ Emad E. Mahmoud $\left(\mathbb{D},{ }^{1}\right.$ and M. Higazy ${ }^{1}$ \\ ${ }^{1}$ Department of Mathematics and Statistics, College of Science, Taif University, P.O. Box 11099, Taif 21944, Saudi Arabia \\ ${ }^{2}$ Department of Mathematics, Faculty of Science, South Valley University, Qena 83523, Egypt \\ ${ }^{3}$ Department of Computer Science, Faculty of Computers and Information, Luxor University, Luxor, Egypt
}

Correspondence should be addressed to S. M. Abo-Dahab; sdahb@yahoo.com

Received 11 January 2021; Revised 16 January 2021; Accepted 21 January 2021; Published 30 January 2021

Academic Editor: Ahmed Mostafa Khalil

Copyright (c) 2021 Hammad Alotaibi et al. This is an open access article distributed under the Creative Commons Attribution License, which permits unrestricted use, distribution, and reproduction in any medium, provided the original work is properly cited.

\begin{abstract}
The aim of this investigation is making mathematical model for the variation in laser pulse, rotational gravity, and magnetic fields on the generalized thermoelastic homogeneous isotropic half-space. The governing dynamical system equations have been formulated considering the four thermoelastic models: coupled (CT) model, Lord and Shulman (LS) model, Green and Lindsay (GL) theory, and Green and Naghdi (GN III) model. Normal mode analysis technique is used to obtain the analytical expressions for the displacement components, temperature, and mechanical and Maxwell's stresses distribution. The effect of laser pulse, gravity, and magnetic field is studied by numerical examples and displayed graphically. A comparison has been made between the theories as well as the present results and agreement with it as a special case from this study. The results predict the strong effect of magnetic field, laser pulse, and gravity field on the wave propagation phenomenon.
\end{abstract}

\section{Introduction}

In last year, the magnetic field, thermal field, elastic field, and rotation interaction received more attention due to the various applications in astronomy, geophysics, engineering, structures, medicine, etc. The topic of thermoelasticity with magnetic field has received the attention of many researchers because of the applications, especially the practical in labs. Biot [1] is the first to discuss the developed thermoelastic theories to overcome the propagation of thermal signals in infinite speed as predicted by the classical thermoelastic coupled dynamical theory. The theories of generalized thermoelasticity have been developed to remove the confliction of the infinite speed of thermal signals in coupled thermoelasticity, which is a physically impossible phenomenon (see [2, 3]). Green et al. [4, 5] formulated three models of thermoelasticity named GN (types I, II, and III).
The gravitational effect on the wave propagation in solids in an elastic globe was investigated by Bromwich [6]. Othman et al. [7] investigated the rotation and gravitational effect on the generalized thermoelastic medium in the context of model of a dual-phase lag. The surface wave propagation in a nonhomogeneous medium under the parameter of gravitational is investigated by Das et al. [8]. Abd-Alla et al. [9] discussed the thermoelastic wave propagation in an isotropic homogeneous half-space of a material under gravity field. The Stoneley, Love, and Rayleigh waves in anisotropic fibrereinforced general viscoelastic media of higher order have been pointed out [10] considering the rotational effect. The Earth's material nature generally is magnetoelastic which may affect the propagation of waves. The thermal stress and magnetic field effect in thermoelasticity neglecting the dissipation of the energy is pointed out by Abo-Dahab et al. [11]. Abo-Dahab et al. [12] pointed out S-waves propagation 
in anisotropic nonhomogeneous medium with magnetic field initial stress, gravity field, and rotation. Some recent works on two-temperature effect with or without rotation are discussed in $[13,14]$. Recently, Abo-Dahab et al. [15] investigated the two-dimensional magnetic field and rotation in generalized thermoelasticity. Lotfy et al. [16] introduced the problem of semiconducting response in an infinite medium with two-temperature and photothermal excitation because of the laser pulse. Abo-Dahab [17] discussed the generalized magneto-thermoelastic reflection waves under two-temperature, thermal shock, and initial stress. Recently, Saroj et al. [18] investigated the Love waves transference in prestressed PZT-5H material stick. The authors in $[19,20]$ discussed waves propagation considering new external parameters and types of waves. Abo-Dahab et al. [21] investigated the problem of magneto-thermoelasticity under influence of laser pulse and gravity field in the context of four theories. The authors in $[22,23]$ arrived to new results considering the magnetization and nonlinear heat on the flow phenomenon. Othman et al. [24] discussed the thermal loading due to laser pulse influence on a generalized micropolar thermoelastic solid with different theories.

In this paper, we suggest mathematical modeling considering the effect of variation in laser pulse, magnetic field gravity, and rotation on a generalized thermoelastic model in an isotropic homogeneous half-space. The governing dynamical system equations have been formulated considering the four thermoelastic models. The four thermoelastic theories are coupled (CT), Lord and Shulman (LS), Green and Lindsay (GL), and Green and Naghdi (GN III) theories. The technique of normal mode technique is used to analyze the expressions for the displacements, temperature, and mechanical and Maxwell's stresses. The effect of gravity, rotation, laser pulse, and magnetic field is studied and displayed graphically. A comparison has been made between the theories as well as the present results and the previous results concluded by the othersand agreement with it as a special case from this study. The results obtained have a significant rule in engineering, astronomy, aircrafts, dynamical system reactors, and aircrafts.

\section{Formulation of the Problem and Basic Equations}

A coordinate of Cartesian system $(x, y, z)$ is considered on the surface $y=0$ and pointing $z$-axis vertically into the halfspace medium $(x \geq 0)$, as shown in Figure 1 .

The dynamic displacement vector in two dimension $u=(u, 0, w)$, and consider all the quantities are dependent on $(t, x, z)$.

The fundamental equations of linear rotational generalized thermoelasticity, where $\vec{\Omega}=(0, \Omega, 0)$ considering Coriolis component and magnetic field $\vec{H}=\left(0, H_{o}, 0\right)$ in the absence of heat sources, take the following form:

$$
\begin{aligned}
\mu u_{i, j j}+(\lambda+\mu) u_{j, i j}-\gamma\left(1+\vartheta_{0} \frac{\partial}{\partial t}\right) T_{, i}++F_{i}+G_{i} & =\rho\left[\frac{\partial^{2} u}{\partial t^{2}}-\Omega^{2} u\right] \\
k T_{, i i}+k^{*} \dot{T}_{, i i} & =\rho C_{e}\left(n_{1} \frac{\partial}{\partial t}+\tau_{0} \frac{\partial^{2}}{\partial t^{2}}\right) T+\gamma T_{0}\left(n_{1} \frac{\partial}{\partial t}+n_{0} \tau_{0} \frac{\partial^{2}}{\partial t^{2}}\right)(\nabla \cdot u)-\rho \dot{Q}, \\
\sigma_{i j} & =\left[\lambda u_{k, k}-\left(1+\vartheta_{0} \frac{\partial}{\partial t}\right) T\right] \delta_{i j}+2 \mu e_{i j}, \quad i, j, k=1,2,3 \\
e_{i j} & =\frac{1}{2}\left(u_{i, j}+u_{j, i}\right), \quad i, j=1,2,3 .
\end{aligned}
$$

The laser pulse is given as

$$
Q=\frac{I_{0} \bar{\gamma}}{2 \pi r^{2}} \exp \left(-\frac{z^{2}}{r^{2}}-\bar{\gamma} x\right) f(t) .
$$

The temporal function $f(t)$ takes the following form:

$$
f(t)=\frac{t}{t_{0}^{2}} \exp \left(-\frac{t}{t_{0}}\right) .
$$

Assuming a homogeneous, thermally, and conducting electrically elastic solid, the electrodynamics system linear equations of slowly moving in a medium are

$$
\begin{aligned}
\operatorname{curl} \vec{h} & =\vec{J}, \\
\operatorname{curl} \vec{E} & =-\mu_{0} \vec{h}, \\
\operatorname{div} \vec{h} & =0, \\
\operatorname{div} \vec{E} & =0, \\
\vec{E} & =-\mu_{0}(\vec{u} \times \vec{H}) .
\end{aligned}
$$

Considering the laser pulse and gravitational field, the governing fundamental equations of a linear homogeneous isotropic thermoelastic medium take the following form: 


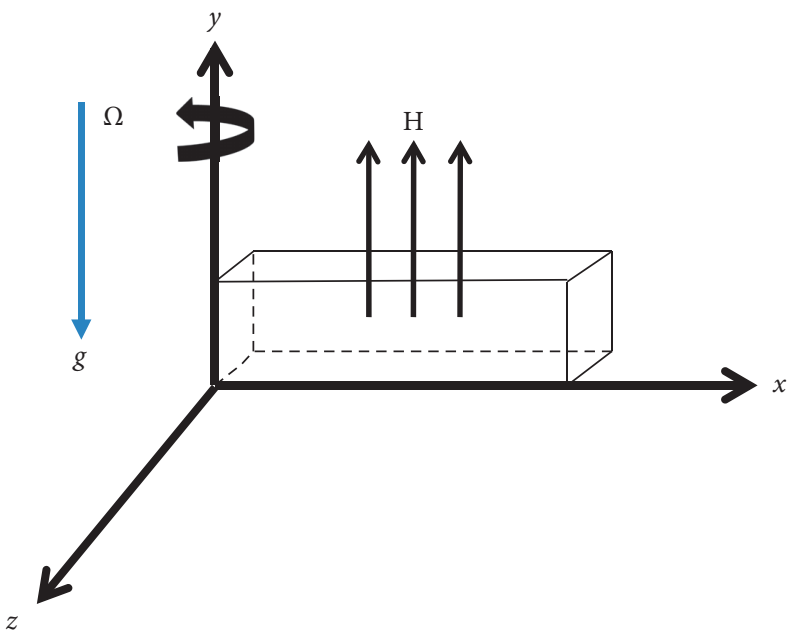

Figure 1: Schematic of the problem.

$$
\begin{aligned}
& \mu \nabla^{2} u+(\lambda+\mu) \frac{\partial e}{\partial x}-\gamma\left(1+\vartheta_{0} \frac{\partial}{\partial t}\right) \frac{\partial T}{\partial x}+\rho g \frac{\partial w}{\partial x}-\mu_{0} H_{0} \frac{\partial h}{\partial x}=\rho\left[\frac{\partial^{2} u}{\partial t^{2}}-\Omega^{2} u\right] \\
& \mu \nabla^{2} w+(\lambda+\mu) \frac{\partial e}{\partial z}-\gamma\left(1+\vartheta_{0} \frac{\partial}{\partial t}\right) \frac{\partial T}{\partial z}-\rho g \frac{\partial u}{\partial x}-\mu_{0} H_{0} \frac{\partial h}{\partial z}=\rho\left[\frac{\partial^{2} w}{\partial t^{2}}-\Omega^{2} w\right] \\
& k \nabla^{2} T+k^{*} \frac{\partial}{\partial t} \nabla^{2} T=\rho C_{e}\left(n_{1} \frac{\partial}{\partial t}+\tau_{0} \frac{\partial^{2}}{\partial t^{2}}\right) T+\gamma T_{0}\left(n_{1} \frac{\partial}{\partial t}+n_{0} \tau_{0} \frac{\partial^{2}}{\partial t^{2}}\right)(\nabla \cdot u)-\rho \frac{\partial Q}{\partial t} .
\end{aligned}
$$

In the context of the thermoelastic models, equations (5)-(7) are applicable to the following:

(i) CT model: $n_{0}=n_{1}=1, \tau_{0}=\vartheta_{0}=0, k^{*}=0$

(ii) L-S model: $n_{0}=n_{1}=1, \tau_{0}>0, \vartheta_{0}=0, k^{*}=0$

(iii) G-L model: $n_{0}=0, n_{1}=1, \vartheta_{0} \geq \tau_{0}>0, k^{*}=0$

(iv) G-N II model: $n_{0}=1, n_{1}=\vartheta_{0}=0, \tau_{0}=1, k^{*}>0$

We know that the CT model considered the coupling between the thermal and strain; assume one and two relaxation times for L-S and G-L models, respectively, but take into account absence of energy dissipation for G-N II.

The previous quantities in the dimensionless variables are as follows:

$$
\begin{aligned}
\left\{x^{\prime}, z^{\prime}\right\} & =\frac{\omega^{*}}{c_{0}}\{x, z\}, \\
\vartheta_{0}^{\prime} & =\omega^{*} \vartheta_{0}, \\
t^{\prime} & =\omega^{*} t, \\
\tau_{0}^{\prime} & =\omega^{*} \tau, \\
Q^{\prime} & =\frac{Q}{w^{*} T_{0} C_{e}},
\end{aligned}
$$

$$
\begin{aligned}
\Omega^{\prime} & =\frac{\Omega}{\omega^{*}}, \\
\left\{u^{\prime}, w^{\prime}\right\} & =\frac{\rho c_{0} \omega^{*}}{v T_{0}}\{u, w\}, \\
T^{\prime} & =\frac{T}{T_{0}}, \\
\delta_{i j}^{\prime} & =\frac{\delta_{i j}}{v T_{0}}, \\
g^{\prime} & =\frac{g}{c_{0} w^{*}}, \\
h^{\prime} & =\frac{h}{H_{0}},
\end{aligned}
$$

where

$$
\begin{gathered}
\omega^{*}=\frac{\rho C_{E} c_{0}^{2}}{K}, \\
c_{0}^{2}=\frac{\lambda+2 \mu}{\rho} .
\end{gathered}
$$

Equations (5)-(7) in the nondimensional form with dropping primes for convenience take the following form: 


$$
\begin{aligned}
& \nabla^{2} u+b_{1} \frac{\partial e}{\partial x}-b_{2}\left(1+\vartheta_{0} \frac{\partial}{\partial t}\right) \frac{\partial T}{\partial x}+b_{3} \frac{\partial w}{\partial x}-R_{h} \frac{\partial h}{\partial x}=b_{2}\left[\frac{\partial^{2} u}{\partial t^{2}}-\Omega^{2} u\right] \\
& \nabla^{2} w+b_{1} \frac{\partial e}{\partial z}-b_{2}\left(1+\vartheta_{0} \frac{\partial}{\partial t}\right) \frac{\partial T}{\partial z}-b_{3} \frac{\partial u}{\partial x}-R_{h} \frac{\partial h}{\partial z}=b_{2}\left[\frac{\partial^{2} w}{\partial t^{2}}-\Omega^{2} w\right] \\
& \varepsilon_{3} \nabla^{2} T+\varepsilon_{2} \frac{\partial}{\partial t} \nabla^{2} T=\varepsilon_{4}\left(n_{1} \frac{\partial}{\partial t}+\tau_{0} \omega^{*} \frac{\partial^{2}}{\partial t^{2}}\right) T+\varepsilon_{1}\left(n_{1} \frac{\partial}{\partial t}+n_{0} \tau_{0} \frac{\partial^{2}}{\partial t^{2}}\right) e-\frac{\partial Q}{\partial t}
\end{aligned}
$$

where

$$
\begin{aligned}
& \varepsilon_{1}=\frac{\gamma^{2} T_{0}}{w^{*} c_{0}^{2} \rho C_{e}}, \\
& \varepsilon_{2}=\frac{k^{*} w^{*}}{\rho c_{0}^{2} C_{e}}, \\
& \varepsilon_{3}=\frac{k}{\rho c_{0}^{2} C_{e}}, \\
& \varepsilon_{4}=\frac{1}{\omega^{*}}, \\
& b_{1}=\frac{\lambda+\mu}{\mu}, \\
& b_{2}=\frac{\rho c_{0}^{2}}{\mu}, \\
& R_{h}=\frac{\mu_{0} H_{0}^{2}}{\mu} . \\
& b_{3}=\frac{\rho g c_{0}^{2}}{\mu},
\end{aligned}
$$

The components of displacement $u(x, z, t)$ and $w(x, z, t)$ considering the rigid body take each of the functions of potential $\psi_{1}(x, z, t)$ and $\psi_{2}(x, z, t)$ in the dimensionless forms as follows:

$$
\begin{aligned}
& u=\frac{\partial \psi_{1}}{\partial x}-\frac{\partial \psi_{2}}{\partial z} \\
& w=\frac{\partial \psi_{1}}{\partial z}+\frac{\partial \psi_{2}}{\partial x}
\end{aligned}
$$

with

$$
\begin{aligned}
e & =\nabla^{2} \psi_{1}, \\
\left(\frac{\partial u}{\partial z}-\frac{\partial w}{\partial x}\right) & =\nabla^{2} \psi_{2}
\end{aligned}
$$

Using (14) and (15) into (10)-(12), we get

$$
\begin{gathered}
{\left[\left(1+b_{1}-R_{h}\right) \nabla^{2}-b_{2} \frac{\partial^{2}}{\partial t^{2}}-b_{2} \Omega^{2}\right] \psi_{1}+b_{3} \frac{\partial}{\partial x} \psi_{2}-b_{2}\left(1+\theta_{0} \frac{\partial}{\partial t}\right) T=0} \\
-b_{3} \frac{\partial}{\partial x} \psi_{1}+\left[\nabla^{2}-b_{2} \frac{\partial^{2}}{\partial t^{2}}+b_{2} \Omega^{2}\right] \psi_{2}=0, \\
-\varepsilon_{1}\left(\frac{n_{1}}{\omega^{*}} \frac{\partial}{\partial t}+n_{0} \tau_{0} \frac{\partial^{2}}{\partial t^{2}}\right) \nabla^{2} \psi_{1}+\left(\varepsilon_{3}+\varepsilon_{2} \frac{\partial}{\partial t}\right) \nabla^{2} T-\varepsilon_{4}\left(n_{1} \frac{\partial}{\partial t}+\tau_{0} \omega^{*} \frac{\partial^{2}}{\partial t^{2}}\right) T=-\frac{\partial}{\partial t} Q .
\end{gathered}
$$

The stress-strain (constitutive) relation gives the following form:

$$
\sigma_{x x}=\frac{\partial u}{\partial x}+L \frac{\partial w}{\partial z}-\left(1+\vartheta_{0} \frac{\partial}{\partial t}\right) T
$$




$$
\begin{aligned}
\sigma_{y y} & =L e-\left(1+\vartheta_{0} \frac{\partial}{\partial t}\right) T \\
\sigma_{z z} & =\frac{\partial w}{\partial z}+L \frac{\partial u}{\partial x}-\left(1+\vartheta_{0} \frac{\partial}{\partial t}\right) T \\
\sigma_{x z} & =\frac{1}{b_{2}}\left(\frac{\partial u}{\partial z}+\frac{\partial w}{\partial x}\right), \quad \sigma_{x y}=\sigma_{y z}=0 . \\
\tau_{z z} & =\bar{G}\left(\frac{\partial u}{\partial x}+\frac{\partial w}{\partial z}\right)
\end{aligned}
$$

where

$$
\begin{gathered}
L=\frac{\lambda}{\lambda+2 \mu}, \\
\bar{G}=\frac{\mu_{e} H_{0}^{2}}{\lambda+2 \mu} .
\end{gathered}
$$

\section{Technique of Normal Mode Analysis}

The physical quantities solution in terms of the normal mode technique as an amplitude functions is multiplied by an exponential function as follows:

$$
\left[\psi_{1}, \psi_{2}, T\right](x, z, t)=\left[\psi_{1}^{*}, \psi_{2}^{*}, T^{*}\right](x) \exp [(\omega t+i a z)],
$$

where $\left[\psi_{1}^{*}, \psi_{2}^{*}, T^{*}\right](x)$ are the physical quantities amplitudes and $i=\sqrt{-1}$. get

Substituting equation (26) into equations (17)-(19), we

$$
\begin{aligned}
{\left[D^{2}-B_{1}\right] \psi_{1}^{*}+B_{2} D \psi_{2}^{*}-B_{3}\left(1+\theta_{0} i \omega\right) T^{*} } & =0 \\
-b_{3} D \psi_{1}^{*}+\left[D^{2}-B_{2}\right] \psi_{2}^{*} & =0 \\
B_{5}\left[D^{2}-a^{2}\right] \psi_{1}^{*}+\left[D^{2}-a^{2}\right] T^{*}-B_{6} T^{*} & =B_{7} \frac{\partial}{\partial t} Q
\end{aligned}
$$

where

$$
\begin{aligned}
& B_{1}=a^{2}-\frac{b_{2} \omega^{2}+b_{2} \Omega^{2}}{1+b_{1}-R_{H}}, \\
& B_{2}=\frac{b_{3}}{1+b_{1}-R_{H}}, \\
& B_{3}=\frac{b_{2}}{1+b_{1}-R_{H}}, \\
& B_{4}=a^{2}-b_{2} \omega^{2}-b_{2} \Omega^{2}, \\
& B_{5}=\frac{\varepsilon_{1} \omega\left(n_{1} i-n_{0} \tau_{0} \omega^{*} \omega\right)}{\left(\varepsilon_{3}+\varepsilon_{2} i \omega\right)} \\
& B_{6}=\frac{-\varepsilon_{4} \omega\left(-n_{1} i+\tau_{0} \omega^{*} \omega\right)}{\left(\varepsilon_{3}+\varepsilon_{2} i \omega\right)}, \\
& B_{7}=\frac{-1}{\left(\varepsilon_{3}+\varepsilon_{2} i \omega\right)}, \\
& D=\frac{d}{\mathrm{~d} x} .
\end{aligned}
$$

Eliminating $\psi_{1}^{*}, \psi_{2}^{*}$, and $T^{*}$ from equations (27)-(29) gives the following differential equations:

$$
\begin{aligned}
& {\left[D^{6}-B_{8} D^{4}+B_{9} D^{2}-B_{10}\right] \psi_{1}^{*}=B_{11}\left(1-\frac{t}{t_{0}}\right) \exp \left[-\left(\frac{z^{2}}{r^{2}}+\frac{t}{t_{0}}+\bar{\gamma} x+i \omega t+i a z\right)\right]} \\
& {\left[D^{6}-B_{8} D^{4}+B_{9} D^{2}-B_{10}\right] T^{*}=B_{12}\left(1-\frac{t}{t_{0}}\right) \exp \left[-\left(\frac{z^{2}}{r^{2}}+\frac{t}{t_{0}}+\bar{\gamma} x+i \omega t+i a z\right)\right]} \\
& {\left[D^{6}-B_{8} D^{4}+B_{9} D^{2}-B_{10}\right] \psi_{2}^{*}=B_{13}\left(1-\frac{t}{t_{0}}\right) \exp \left[-\left(\frac{z^{2}}{r^{2}}+\frac{t}{t_{0}}+\bar{\gamma} x+i \omega t+i a z\right)\right]}
\end{aligned}
$$


where

$$
\begin{aligned}
B_{8}= & B_{1}+B_{4}+B_{6}+B_{2} b_{3}-B_{3} B_{5}-B_{3} B_{5} \vartheta_{0} i+\omega+a^{2}, \\
B_{9}= & a^{2} B_{1}+a^{2} B_{4}+a^{2} B_{2} b_{3}-a^{2} B_{3} B_{5}-B_{3} B_{5} B_{4}+B_{1} B_{4}+B_{6} B_{4}+B_{1} B_{6}+b_{3} B_{2} B_{6} \\
& +B_{3} B_{5} a^{2} i \omega \vartheta_{0}+B_{3} B_{4} B_{5} i \omega \vartheta_{0}, \\
B_{10}= & a^{2} B_{1} B_{4}-a^{2} B_{3} B_{5} B_{4}+B_{1} B_{4} B_{6}-B_{3} B_{4} B_{5} a^{2} i \omega \vartheta_{0}, \\
B_{11}= & B_{3} B_{7}\left(\bar{\gamma}^{2}+i \omega \vartheta_{0} \bar{\gamma}^{2}-B_{4}-B_{4} i \omega \vartheta_{0}\right) \frac{I_{0} \bar{\gamma}}{2 \pi r^{2} t_{0}^{2}} \\
B_{12}= & B_{7}\left[\left(\bar{\gamma}^{2}-B_{1}\right)\left(\bar{\gamma}^{2}-B_{4}\right)+\left(\bar{\gamma}^{2} B_{2} b_{3}\right)\right] \frac{I_{0} \bar{\gamma}}{2 \pi r^{2} t_{0}^{2}} \\
B_{13}= & -B_{3} B_{7} b_{3}\left(1+i \vartheta_{0} \omega\right) \frac{I_{0} \bar{\gamma}^{2}}{2 \pi r^{2} t_{0}^{2}} .
\end{aligned}
$$

Equation (31) can be taken in the following form:

$$
\begin{aligned}
& \left(D^{2}-k_{1}^{2}\right)\left(D^{2}-k_{2}^{2}\right)\left(D^{2}-k_{3}^{2}\right) \psi_{1}^{*} \\
& =B_{11}\left(1-\frac{t}{t_{0}}\right) \exp \left[-\left(\frac{z^{2}}{r^{2}}+\frac{t}{t_{0}}+\bar{\gamma} x+i \omega t+i a z\right)\right],
\end{aligned}
$$

where $k_{n}^{2}(n=1,2,3)$ are the characteristic equation roots of the homogeneous equations (31)-(33).

The general solutions of equations (31)-(33) bounded as $x \longrightarrow \infty$ are given as follows:

$$
\begin{aligned}
& \psi_{1}(x, z, t)=\sum_{n=1}^{3} R_{n} \exp \left(-k_{n} x+i \omega t+i a z\right)+L_{1} B_{11} f_{1} \\
& \psi_{2}(x, z, t)=\sum_{n=1}^{3} H_{1 n} R_{n} \exp \left(-k_{n} x+i \omega t+i a z\right)+L_{1} B_{13} f_{1} \\
& T(x, z, t)=\sum_{n=1}^{3} H_{2 n} R_{n} \exp \left(-k_{n} x+i \omega t+i a z\right)+L_{1} B_{12} f_{1} .
\end{aligned}
$$

Here,

$$
\begin{aligned}
H_{1 n} & =\frac{-b_{3} k_{n}}{\left(k_{n}^{2}-B_{4}\right)}, \quad n=1,2,3, \\
H_{2 n} & =\frac{\left(k_{n}^{2}-B_{1}\right)-B_{2} H_{1 n} k_{n}}{B_{3}}, \quad n=1,2,3, \\
L_{1} & =-\frac{1}{\bar{\gamma}^{6}-B_{8} \bar{\gamma}^{4}+B_{9} \bar{\gamma}^{2}-B_{10}}, \\
f_{1} & =\left(1-\frac{t}{t_{0}}\right) \exp \left(-\frac{z^{2}}{r^{2}}-\frac{t}{t_{0}}-\bar{\gamma} x\right),
\end{aligned}
$$

where $R_{n}(n=1,2,3)$ are the coefficients.

To get the displacement vector components, substituting equations (36) and (37) into equation (14), we obtain

$$
\begin{aligned}
u(x, z, t)= & \sum_{n=1}^{3} M_{1 n} R_{n} \exp \left(-k_{n} x+i \omega t+i a z\right) \\
& -\left(\bar{\gamma} I_{1}+\frac{2 z I_{2}}{r^{2}}\right) \exp \left(-\frac{z^{2}}{r^{2}}-\frac{t}{t_{0}}-\bar{\gamma} x\right), \\
w(x, z, t)= & \sum_{n=1}^{3} M_{2 n} R_{n} \exp \left(-k_{n} x+i \omega t+i a z\right) \\
& +\left(-\bar{\gamma} I_{2}+\frac{2 z I_{1}}{r^{2}}\right) \exp \left(-\frac{z^{2}}{r^{2}}-\frac{t}{t_{0}}-\bar{\gamma} x\right),
\end{aligned}
$$


where $M_{1 n}=-k_{n}-i a H_{1 n}, M_{2 n}=i a-k_{n} H_{1 n}, n=1,2,3$.

To get the stress tensor components, substituting equations (38)-(41) into equations (20)-(24), we get

$$
\begin{aligned}
\sigma_{x x}(x, z, t) & =\sum_{n=1}^{3} H_{3 n} R_{n} \exp \left(-k_{n} x+i \omega t+i a z\right)+I_{4} \exp \left(-\frac{z^{2}}{r^{2}}-\frac{t}{t_{0}}-\bar{\gamma} x\right), \\
\sigma_{y y}(x, z, t) & =\sum_{n=1}^{3} H_{4 n} R_{n} \exp \left(-k_{n} x+i \omega t+i a z\right)+I_{5} \exp \left(-\frac{z^{2}}{r^{2}}-\frac{t}{t_{0}}-\bar{\gamma} x\right), \\
\sigma_{z z}(x, z, t) & =\sum_{n=1}^{3} H_{5 n} R_{n} \exp \left(-k_{n} x+i \omega t+i a z\right)+I_{6} \exp \left(-\frac{z^{2}}{r^{2}}-\frac{t}{t_{0}}-\bar{\gamma} x\right), \\
\sigma_{x z}(x, z, t) & =\sum_{n=1}^{3} H_{6 n} R_{n} \exp \left(-k_{n} x+i \omega t+i a z\right)+I_{7} \exp \left(-\frac{z^{2}}{r^{2}}-\frac{t}{t_{0}}-\bar{\gamma} x\right), \\
\tau_{x x} & =\sum_{n=1}^{3} H_{7 n} \exp \left(-k_{n} x+i \omega t+i a z\right)+I_{8} \exp \left(-\frac{z^{2}}{r^{2}}-\frac{t}{t_{0}}-\bar{\gamma} x\right) .
\end{aligned}
$$

Here,

$$
\begin{aligned}
H_{3 n} & =-M_{1 n} k_{n}+L i a M_{2 n}-H_{2 n}-i \omega \theta_{0} H_{2 n}, \\
H_{4 n} & =-k_{n} M_{1 n} L+i a L M_{2 n}-H_{2 n}-i \omega \theta_{0} H_{2 n}, \\
H_{5 n} & =i a M_{2 n}-L k_{n} M_{1 n}-H_{2 n}-i \omega \theta_{0} H_{2 n}, \\
H_{6 n} & =\frac{1}{b 2}\left(i a M_{1 n}-k_{n} M_{2 n}\right), \\
H_{7 n} & =i a \bar{G} M_{2 n}-k_{n} \bar{G} M_{1 n}, \\
I_{1} & =-B_{10} L_{1}\left(1-\frac{t}{t_{0}}\right), \\
I_{2} & =\frac{B_{12}}{B_{10}} I_{1}, \\
I_{3} & =\frac{B_{11}}{B_{10}} I_{1}, \\
I_{4} & =\bar{\gamma}\left(\bar{\gamma} I_{1} t-n \frac{2 z}{r^{2}} I I_{2}\right)+\frac{2 z}{r^{2}}\left(\bar{\gamma} I_{2} t+n \frac{2 z I_{1}}{r^{2}}\right) L-I_{3}-\frac{\theta_{0}}{t_{0}} I_{3}, \\
I_{5} & =E_{1} \bar{\gamma}\left(\bar{\gamma} I_{1} t-n \frac{2 z I_{2}}{r^{2}}\right)+\frac{2 z}{r^{2}} L\left(\bar{\gamma} I_{2} t+n \frac{2 z I_{1}}{r^{2}}\right)-I_{3}-\frac{\theta_{0}}{t_{0}} I_{3}, \\
I_{6} & =\frac{2 z}{r^{2}}\left(\bar{\gamma} I_{2} t+n \frac{2 z I_{1}}{r^{2}}\right)+L \bar{\gamma}\left(\bar{\gamma} I_{1} t-n \frac{2 z I_{3}}{r^{2}}\right)-I_{3}-\frac{\theta_{0}}{t_{0}} I_{3}, \\
I_{7} & =\frac{1}{b_{2}}\left[\frac{2 z}{r^{2}}\left(\bar{\gamma} I_{1} t-n \frac{2 z I_{3}}{r^{2}}\right)+\bar{\gamma}\left(\bar{\gamma} I_{2} t-n \frac{2 z I_{1}}{r^{2}}\right)\right], \\
I_{8} & \left.=\bar{\gamma} I_{1} t-n \frac{2 z I_{2}}{r^{2}}\right)+\frac{2 z}{r^{2}} \bar{G}\left(\bar{\gamma} I_{2} t+n \frac{2 z I_{1}}{r^{2}}\right) .
\end{aligned}
$$

\section{Boundary Conditions}

We will obtain the constants $R_{n}(n=1,2,3)$, so the boundary conditions considered and should suppress the positive exponentials to avoid them at infinity unboundedness. The chosen coefficients $R_{1}, R_{2}$, and $R_{3}$ will be obtained from the boundary conditions on the surface at $x=0$ as follows.

(i) The mechanical boundary conditions:

$$
\begin{aligned}
\sigma_{z z}+\tau_{z z} & =-p_{1} \exp (\omega t+i a z), \\
\sigma_{x z} & =0 .
\end{aligned}
$$

(ii) On the surface of the half-space, the thermal boundary condition is

$$
\frac{\partial T}{\partial x}=0 .
$$

From the above boundary conditions, we get

$$
\begin{aligned}
\sum_{n=1}^{3}\left(H_{5 n}+H_{7 n}\right) R_{n} & =-p, \\
\sum_{n=1}^{3} H_{6 n} R_{n} & =0, \\
\sum_{n=1}^{3}-k_{n} H_{2 n} R_{n} & =0 .
\end{aligned}
$$

Using the method of matrix, we get the coefficient values of $R_{n}(n=1,2,3)$ : 


$$
\left(\begin{array}{c}
R_{1} \\
R_{2} \\
R_{3}
\end{array}\right)=\left(\begin{array}{ccc}
\left(H_{51}+H_{71}\right) & \left(H_{52}+H_{72}\right) & \left(H_{53}+H_{73}\right) \\
H_{61} & H_{62} & H_{63} \\
-k_{1} H_{21} & -k_{2} H_{22} & -k_{3} H_{23}
\end{array}\right)^{-1}\left(\begin{array}{c}
-p_{1} \\
0 \\
0
\end{array}\right) .
$$

After that, we obtain the displacements, temperatures, and stresses.

\section{Numerical Results and Discussion}

Following Dhaliwal and Singh [25], to calculate the numerical values of the considered variables, the magnesium material was chosen:

$$
\begin{aligned}
\lambda & =2.17 \times 10^{10} \mathrm{~N} / \mathrm{m}^{2}, \\
\mu & =3.278 \times 10^{10} \mathrm{~N} / \mathrm{m}^{2}, \\
K & =1.7 \times 10^{2} \mathrm{~W} / \mathrm{mK}, \\
\rho & =1.74 \times 10^{3} \mathrm{~kg} / \mathrm{m}^{3}, \\
C_{e} & =1.04 \times 10^{3} \mathrm{j} / \mathrm{kgK}, \\
\omega^{*} & =3.58 \times 10^{11} / \mathrm{s}, \\
\mu_{0} & =4 \times \pi \times 10^{-3}, \\
T_{0} & =298 .
\end{aligned}
$$

The laser pulse constants are

$$
\begin{aligned}
I_{0} & =10^{2} \mathrm{j} / \mathrm{m}^{2}, \\
r & =0.2, \\
\bar{\gamma} & =25 / \mathrm{m}, \\
t_{0} & =10 .
\end{aligned}
$$

\section{Consider}

$$
\begin{aligned}
p_{1} & =0.25 \mathrm{~N} / \mathrm{m}^{2}, \\
k^{*} & =100 \mathrm{~W} / \mathrm{mK}, \\
a & =0.5, \\
\omega & =2.9 \mathrm{rad} / \mathrm{s}, \\
z & =2 \mathrm{~m}, \\
t & =0.9 \mathrm{~s}, \\
g & =9.8 \mathrm{~m} / \mathrm{s}^{2}, \quad 0 \leq x \leq 3.5 \mathrm{~m} .
\end{aligned}
$$

Figures 2-33 display the distributions calculated with respect to the range $x(0 \leq x \leq 3.5)$. The variation presented in two-dimensional figures shows the change in behavior of the values of the displacement components $u$ and $w$, stresses $\sigma_{x x}, \sigma_{z z}$, and $\sigma_{x z}$, and also Maxwell's stress and temperature distribution $T$ with distance $x$. In the context of G-N III theory, a schematic has been shown in generalized thermoelasticity medium with constants $H_{0}=9 \times 10^{5}$, $g=9.8$, and $t=0.9$ with different values of gravity, laser pulse, and magnetic field taking into account two values of rotation. Figures 2-9 present a comparison between the variation in the four thermoelastic models: (i) CT theory:

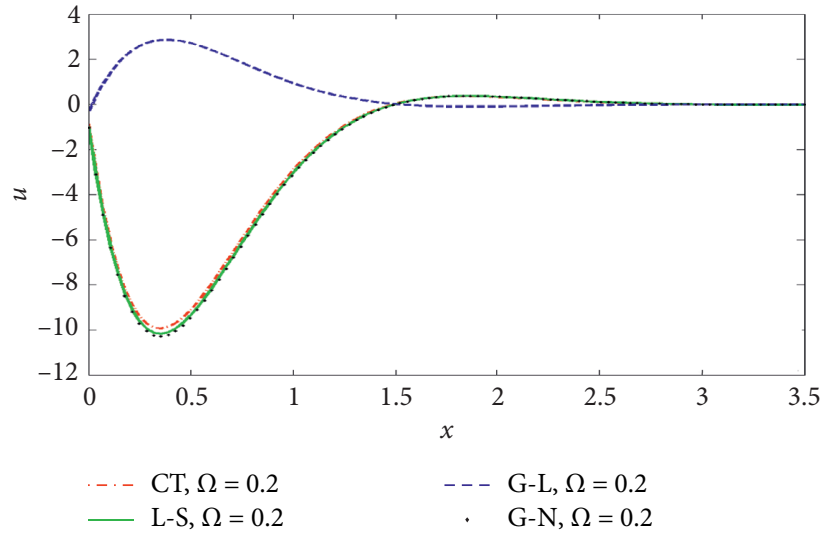

FIgURE 2: Horizontal displacement $u$ concerning $x$ under four thermoelastic theories.

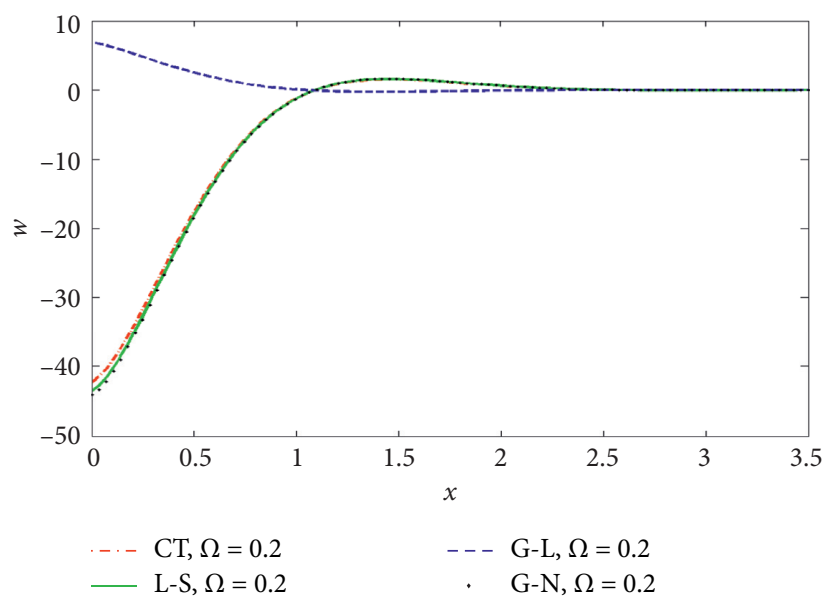

FIGURE 3: Vertical displacement $w$ concerning $x$ under four thermoelastic theories.

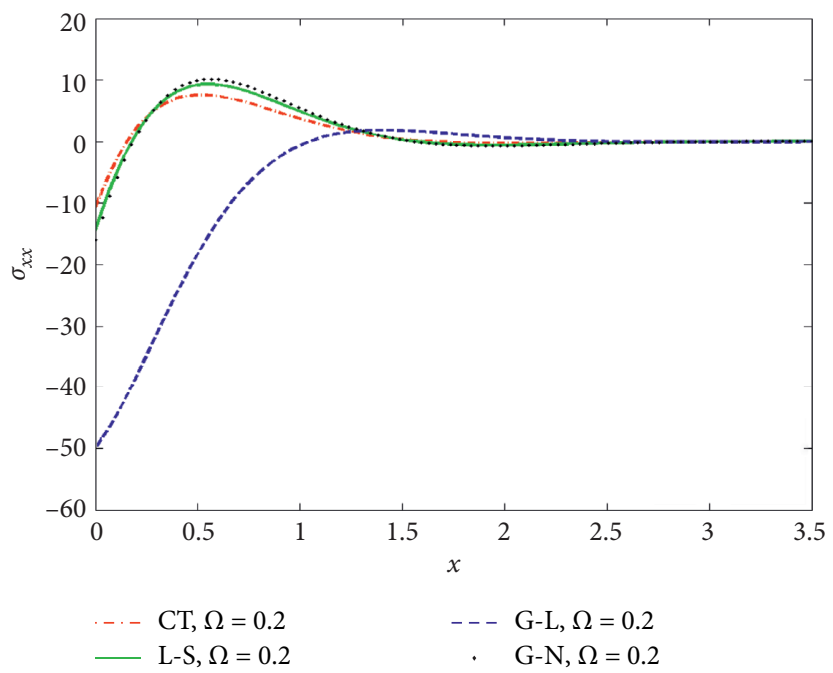

Figure 4: Stress $\sigma_{x x}$ concerning $x$ under four thermoelastic theories.

$n_{0}=0, n_{1}=1, \tau_{0}=0, \vartheta_{0}=0$; (ii) LS theory: $n_{0}=1, n_{1}=$ $1, \tau_{0}=0.2, \vartheta_{0}=0$; (iii) GL theory: $n_{0}=0, n_{1}=1, \tau_{0}=$ $0.2, \vartheta_{0}=0.3$; $\quad$ and (iv) GN theory: $n_{0}=0, n_{1}=1$, 


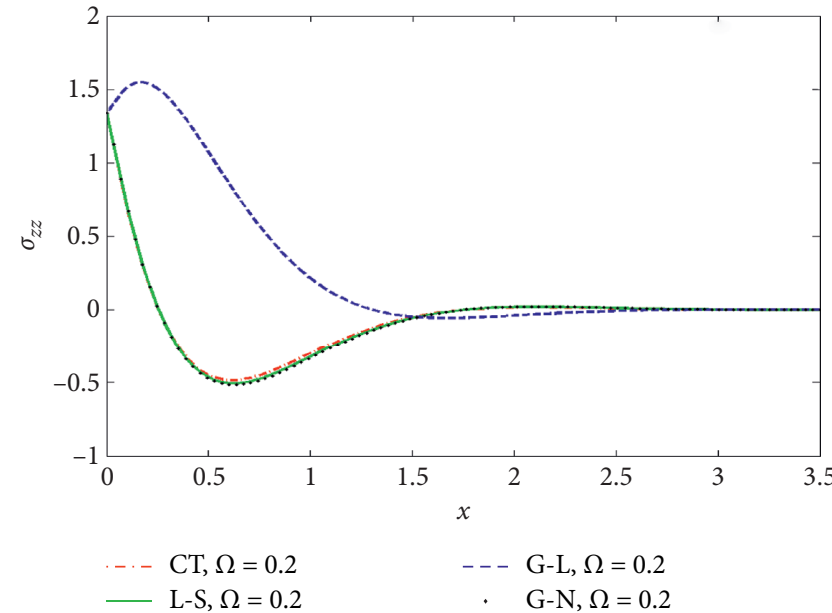

Figure 5: Stress $\sigma_{z z}$ concerning $x$ under four thermoelastic theories.

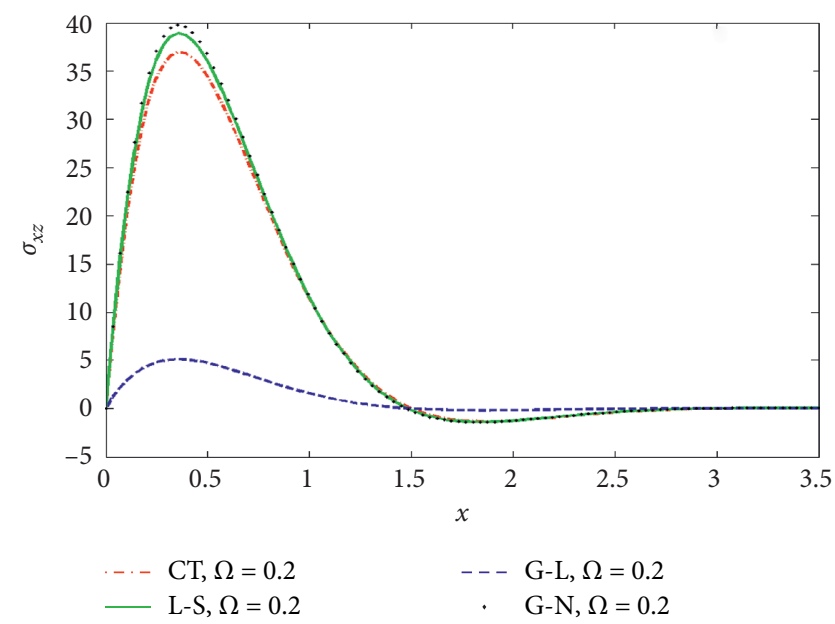

Figure 6: Stress $\sigma_{x z}$ concerning $x$ under four thermoelastic theories.

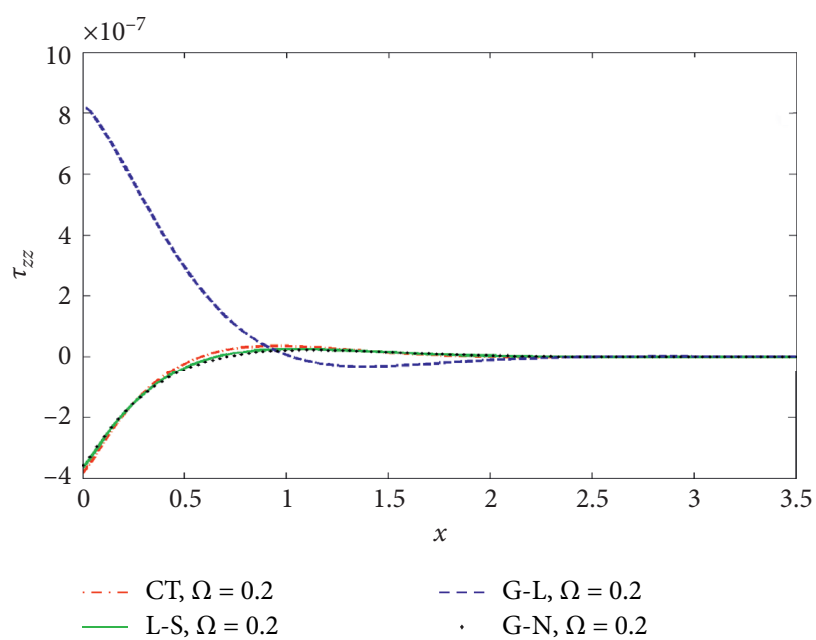

Figure 7: Stress $\tau_{z z}$ concerning $x$ under four thermoelastic theories.

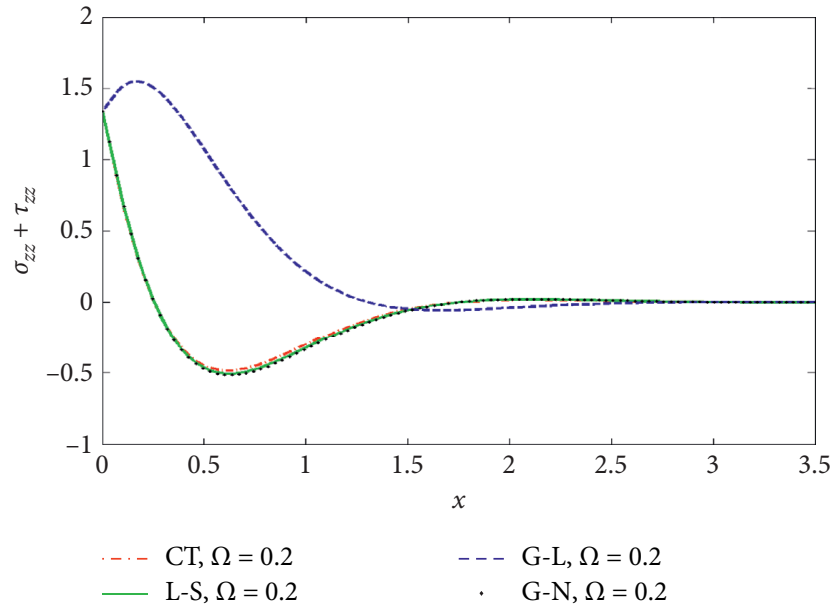

FIGURE 8: Stress $\tau_{z z}+\sigma_{z z}$ concerning $x$ under four thermoelastic theories.

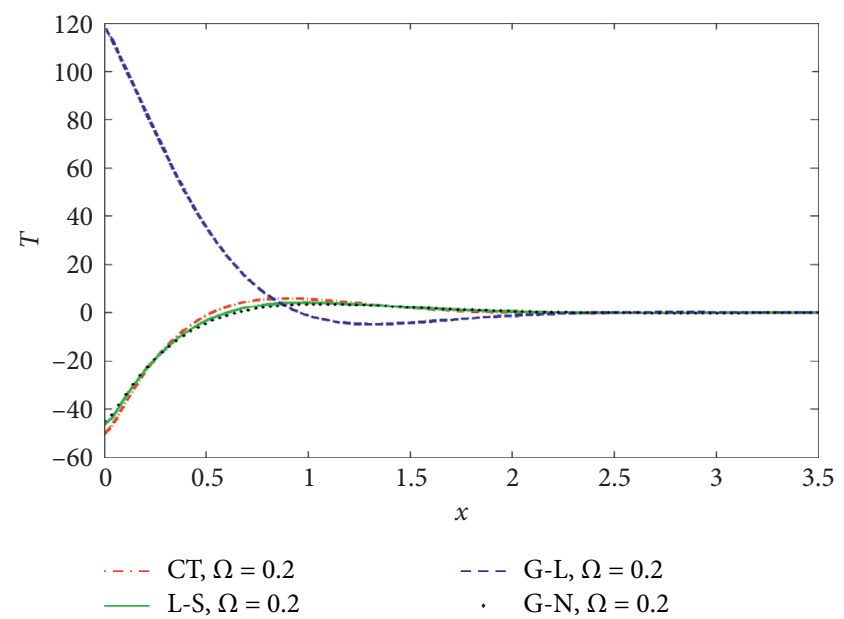

FIgURE 9: Temperature $T$ concerning $x$ under four thermoelastic theories.

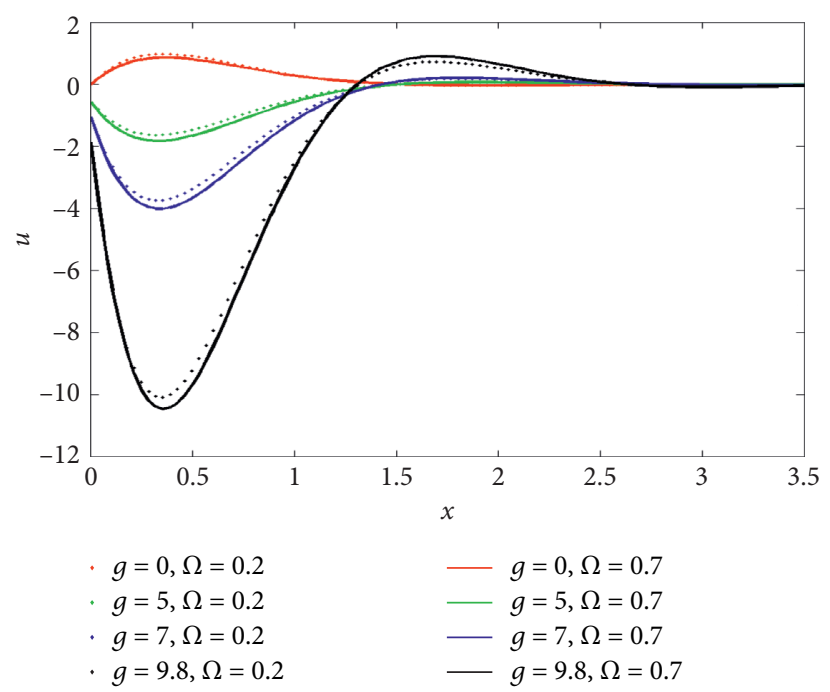

FIGURE 10: Horizontal displacement $u$ concerning $x$ under gravity and rotation. 


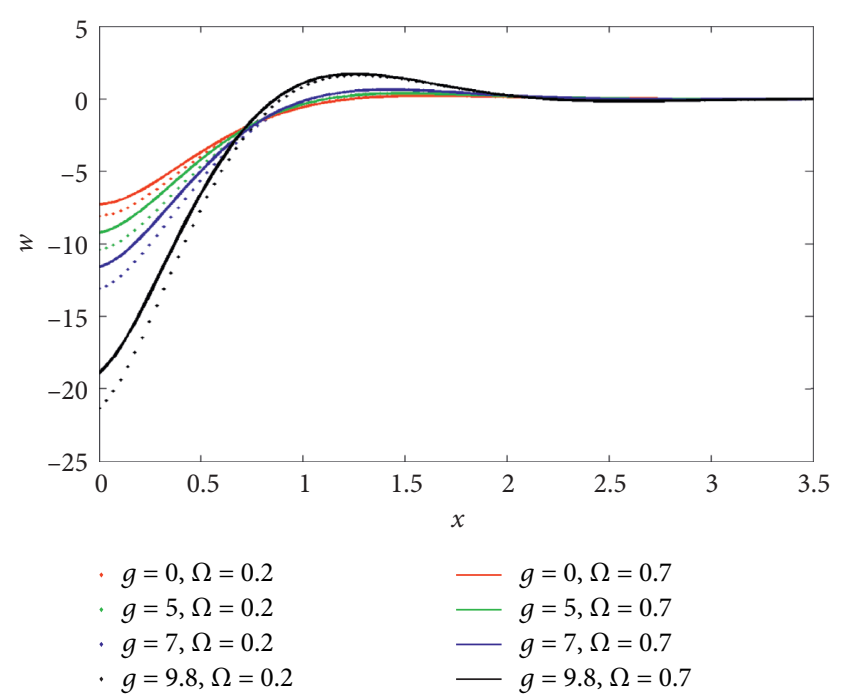

Figure 11: Vertical displacement $w$ concerning $x$ under gravity and rotation.

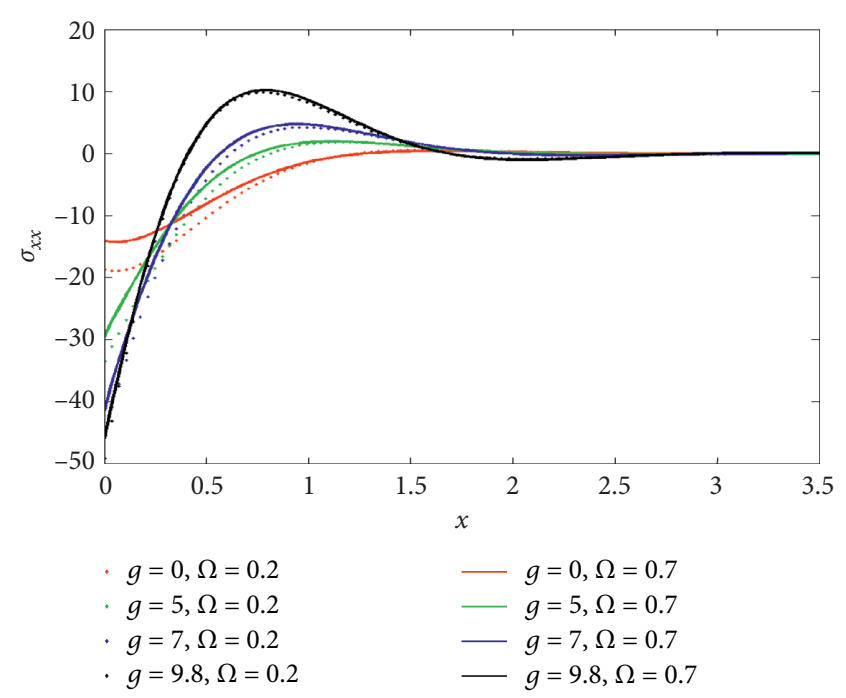

FIgURE 12: Stress $\sigma_{x x}$ concerning $x$ under gravity and rotation.

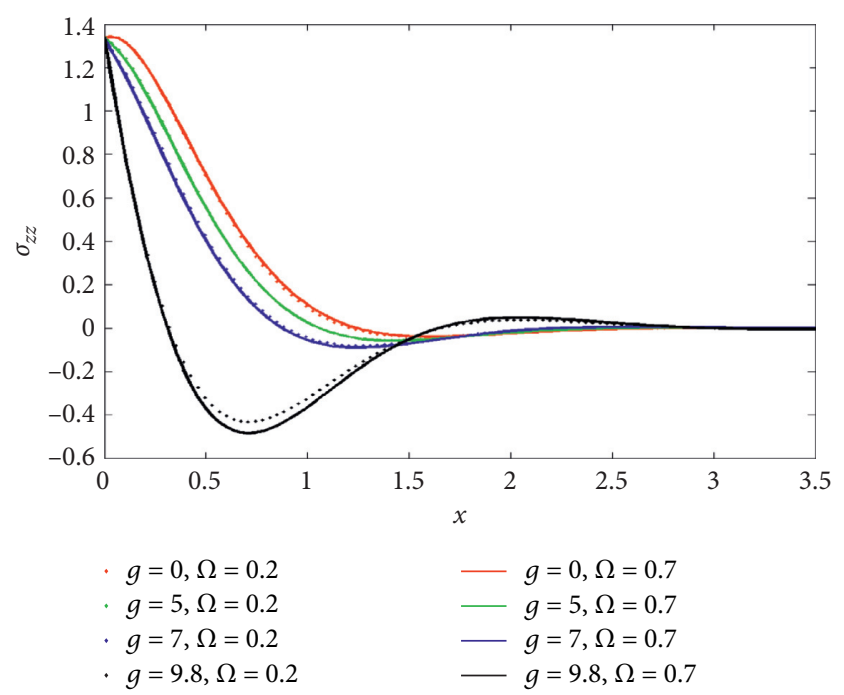

FIGURE 13: Stress $\sigma_{z z}$ concerning $x$ under gravity and rotation.

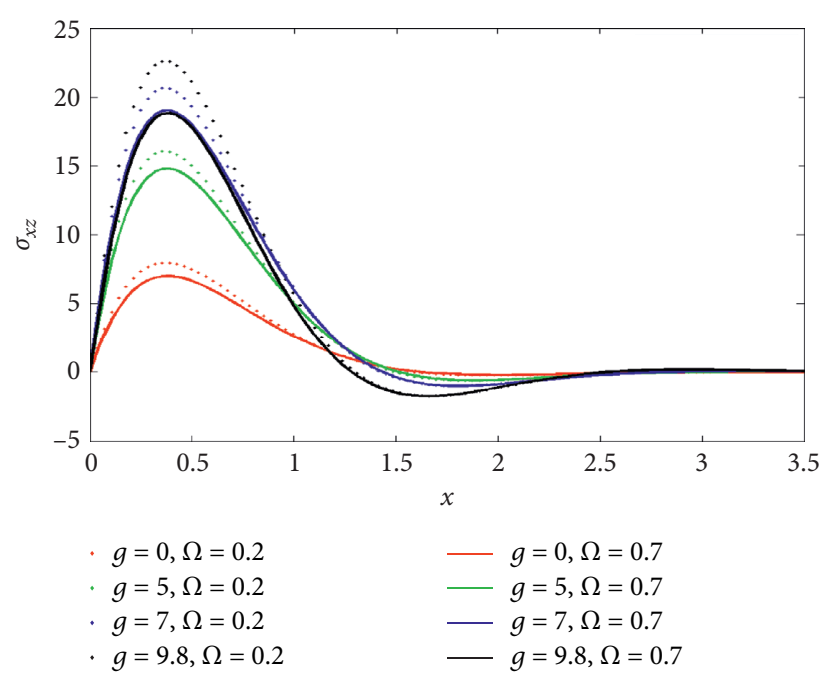

Figure 14: Stress $\sigma_{x z}$ concerning $x$ under gravity and rotation.

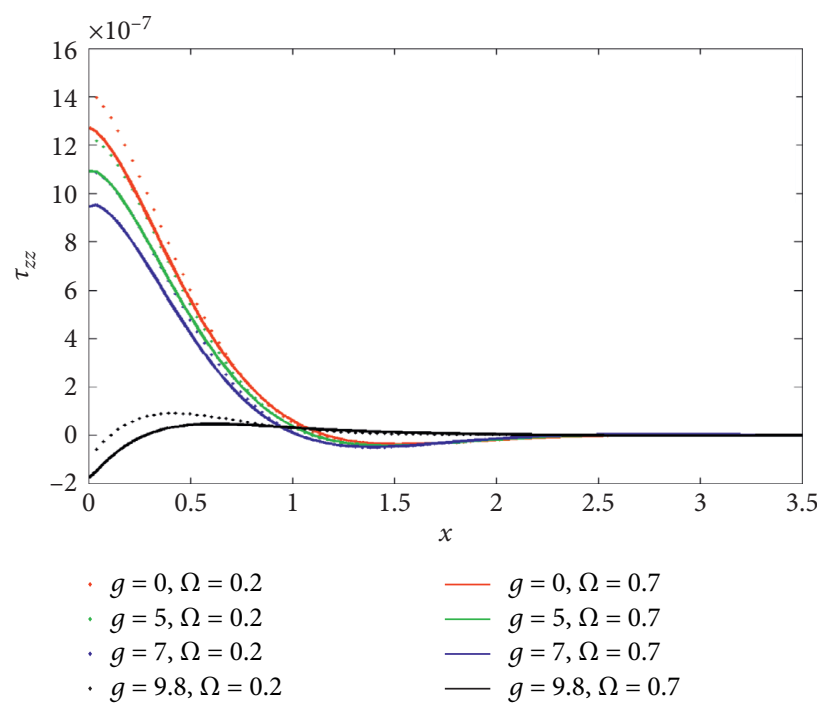

FIGURE 15: Stress $\tau_{z z}$ concerning $x$ under gravity and rotation.

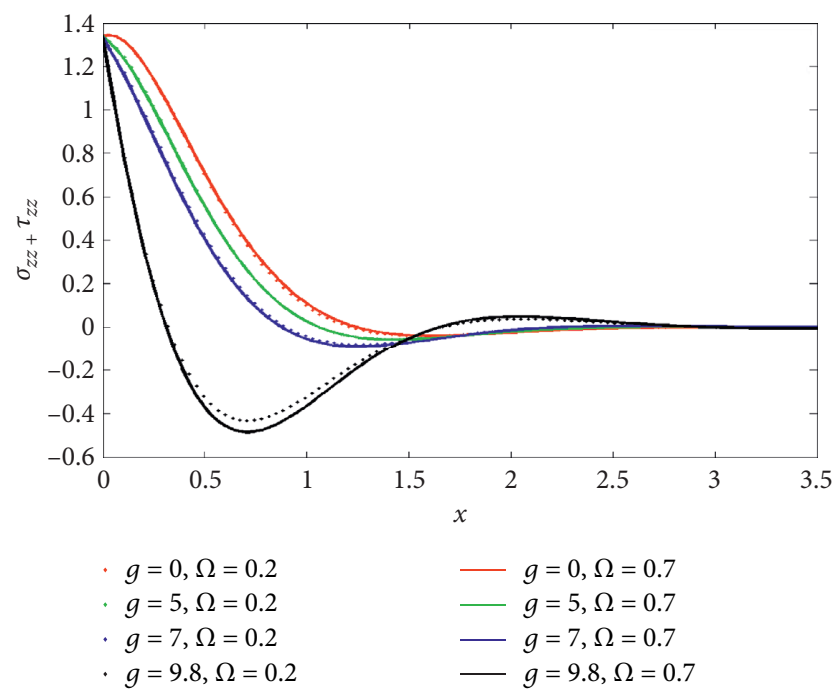

Figure 16: Stress $\tau_{z z}+\sigma_{z z}$ concerning $x$ under gravity and rotation. 


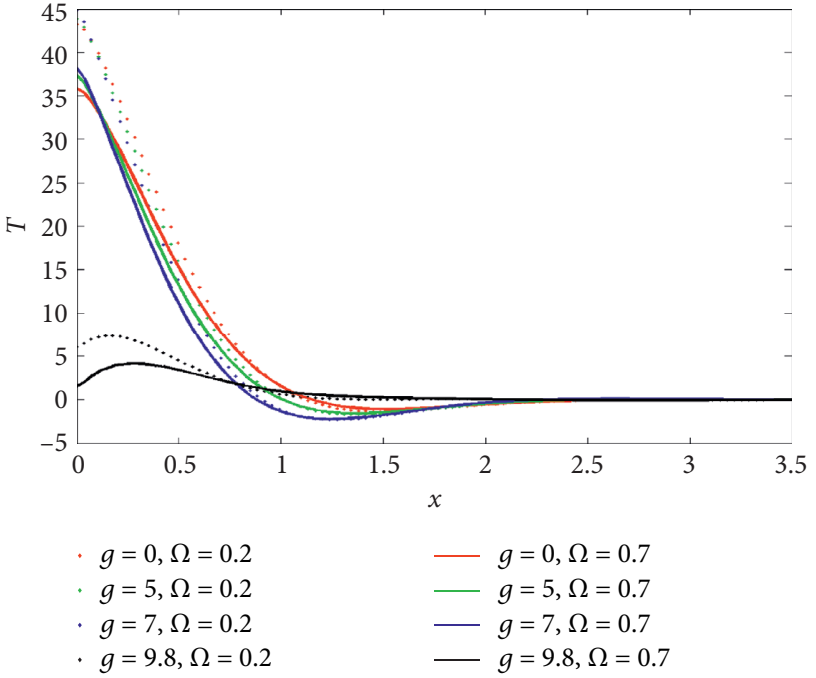

Figure 17: Temperature $T$ concerning $x$ under gravity and rotation.

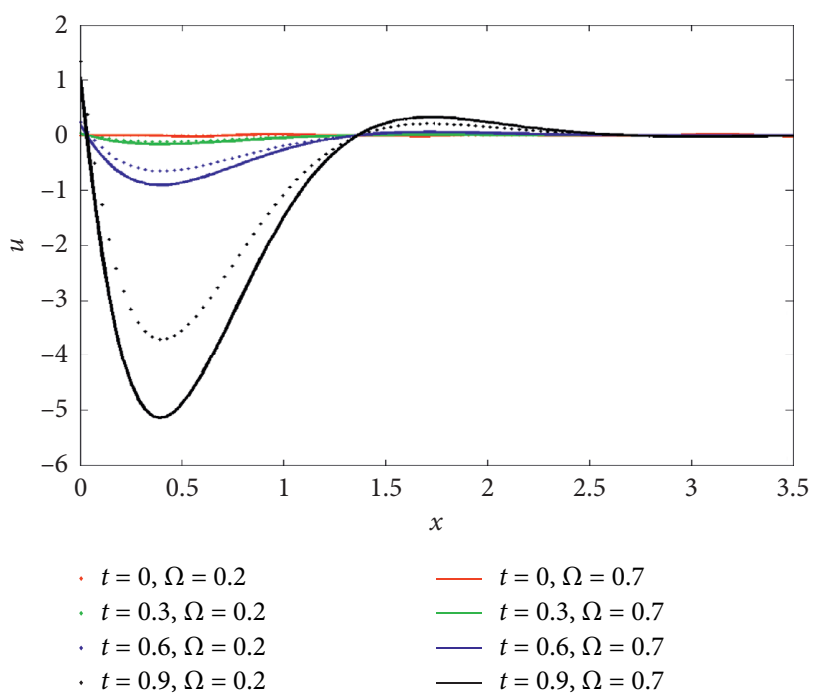

FIGURE 18: Horizontal displacement $u$ concerning $x$ with laser pulse " $t$ " and rotation.

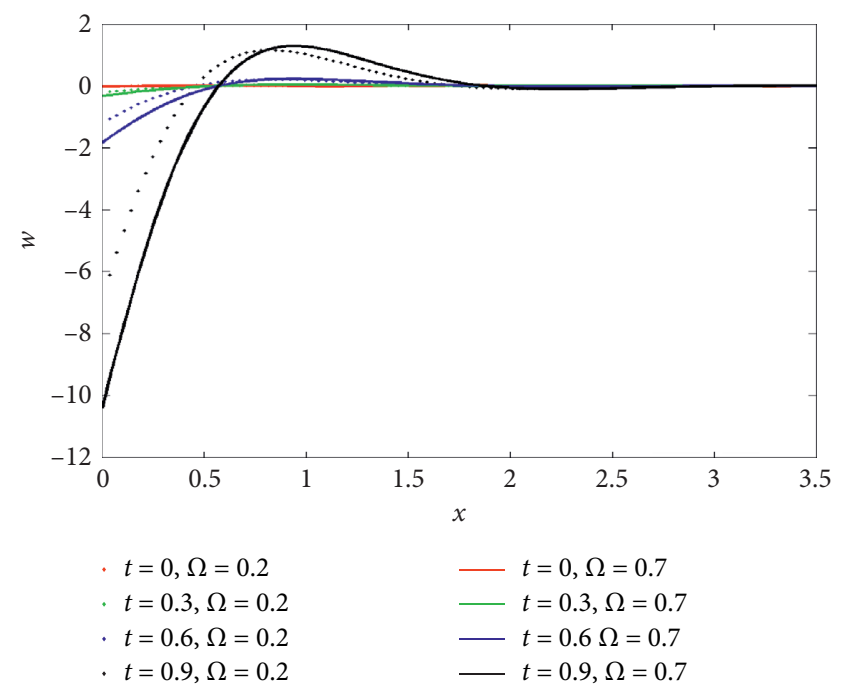

FIGURE 19: Vertical displacement $w$ concerning $x$ with laser pulse " $t$ " and rotation.

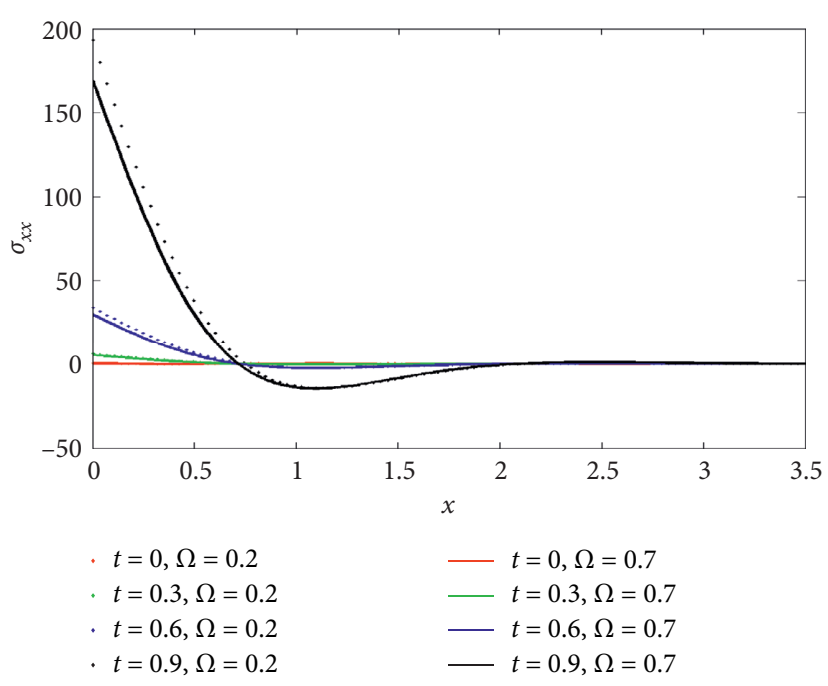

FIgURE 20: Stress $\sigma_{x x}$ concerning $x$ with laser pulse " $t$ " and rotation.

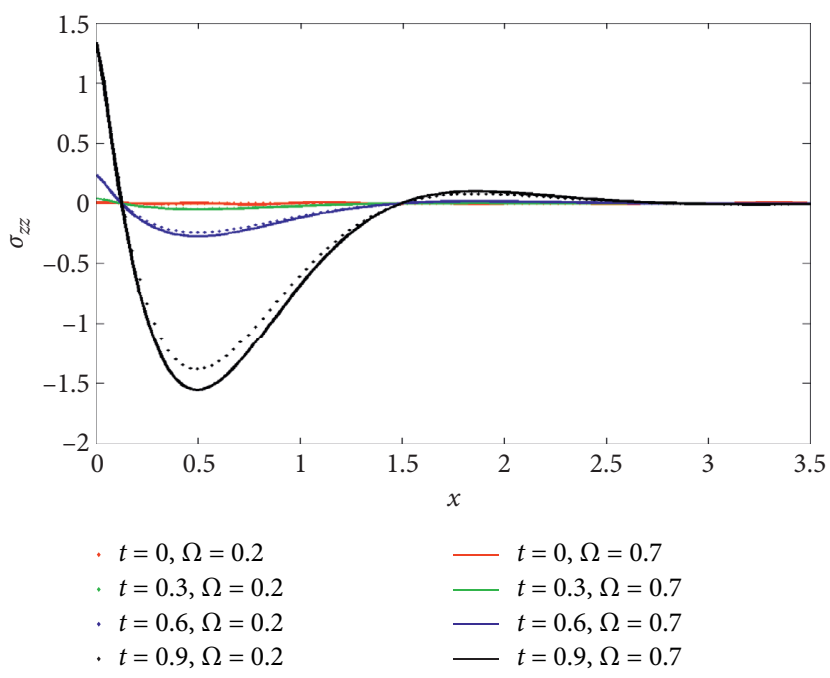

FIgURE 21: Stress $\sigma_{z z}$ concerning $x$ with laser pulse " $t$ " and rotation.

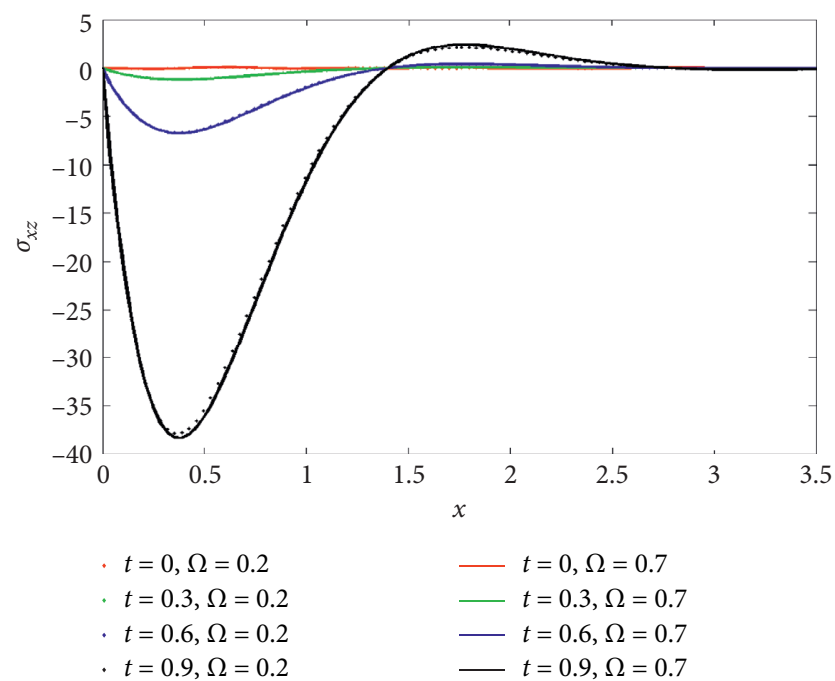

Figure 22: Stress $\sigma_{x z}$ concerning $x$ with laser pulse " $t$ " and rotation. 


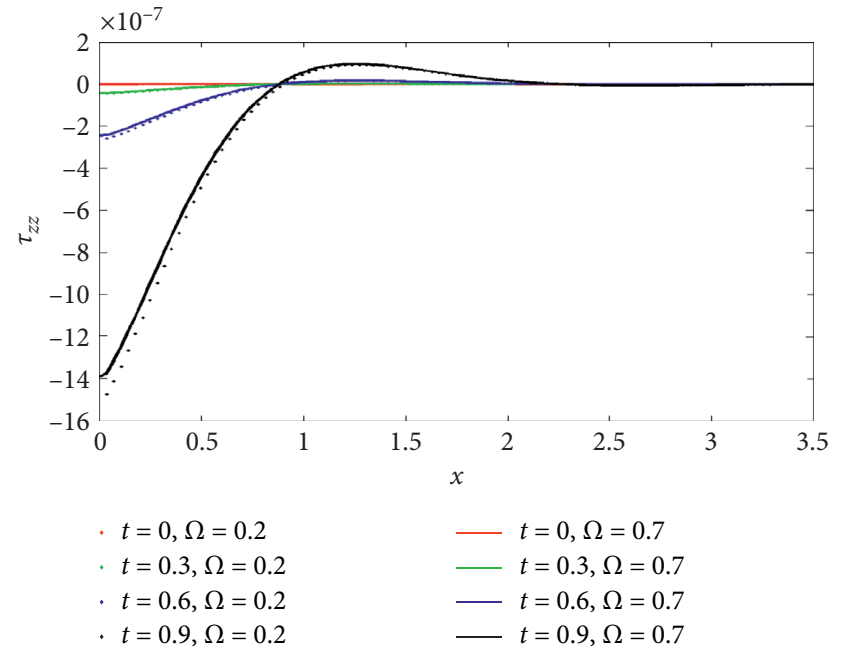

FIGURE 23: Stress $\tau_{z z}$ concerning $x$ with laser pulse " $t$ " and rotation.

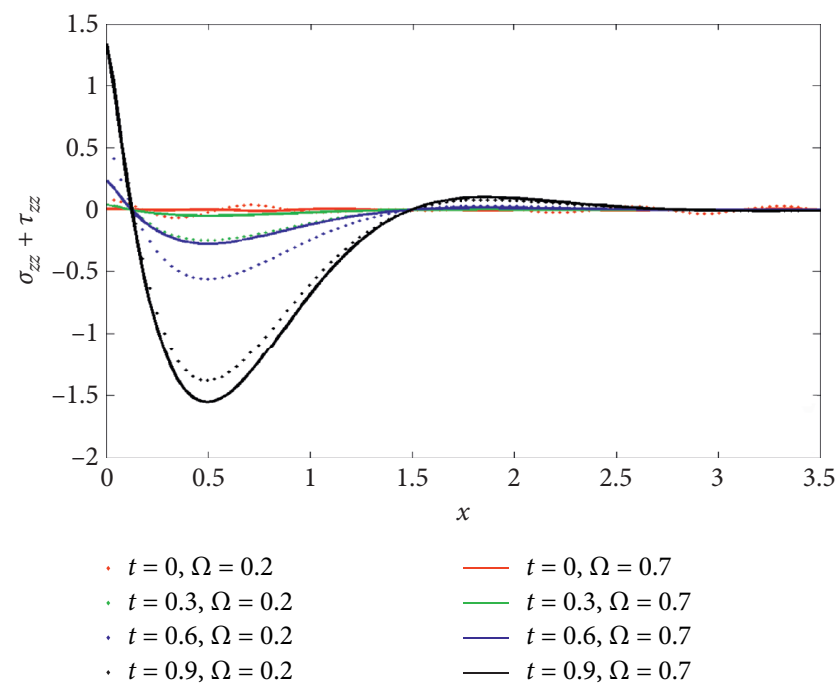

FIgURE 24: Stress $\tau_{z z}+\sigma_{z z}$ concerning $x$ with laser pulse " $t$ " and rotation.

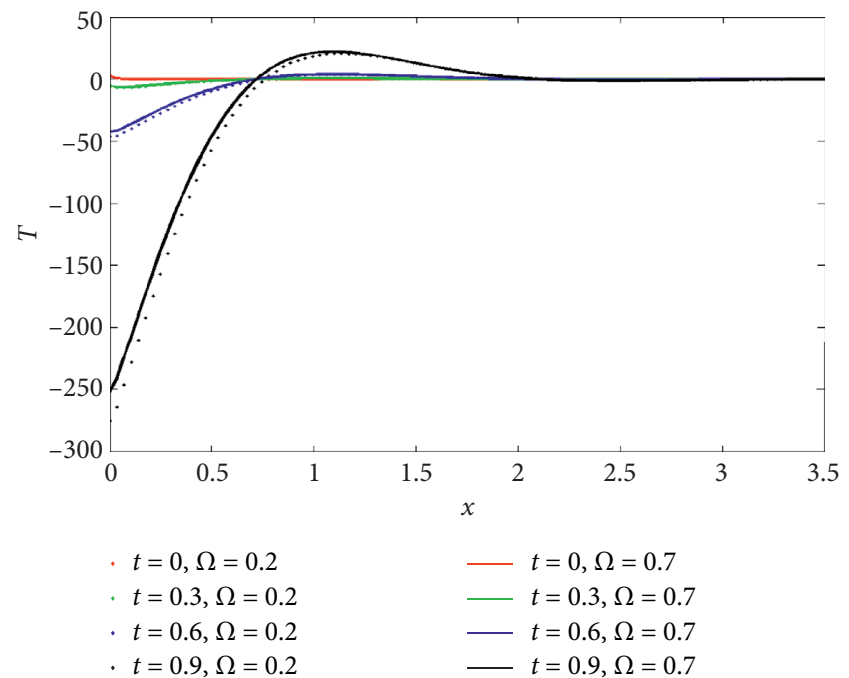

Figure 25: Temperature $T$ concerning $x$ with laser pulse " $t$ " and rotation.

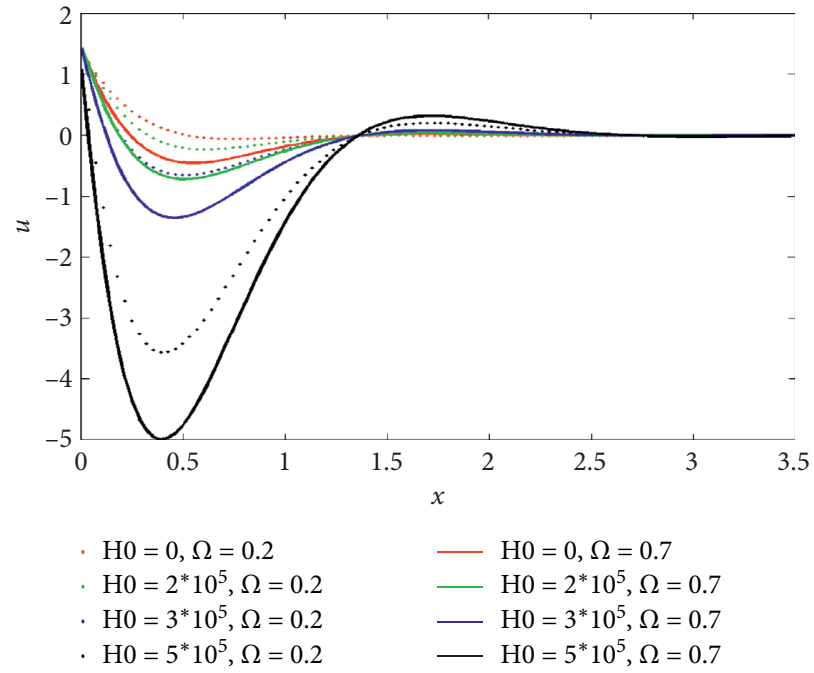

FIgURE 26: Horizontal displacement $u$ concerning $x$ with magnetic and rotation.

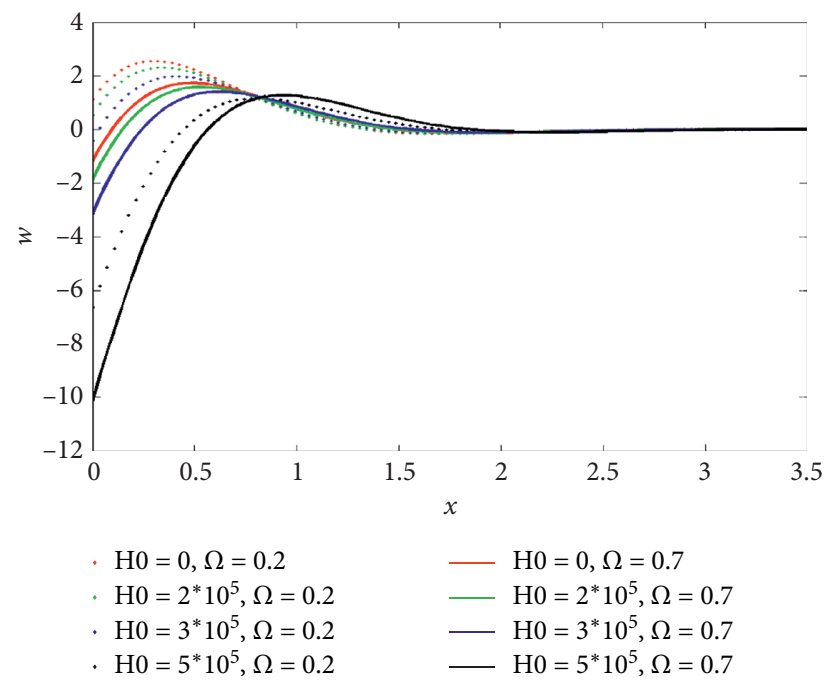

Figure 27: Vertical displacement $w$ concerning $x$ with magnetic and rotation.

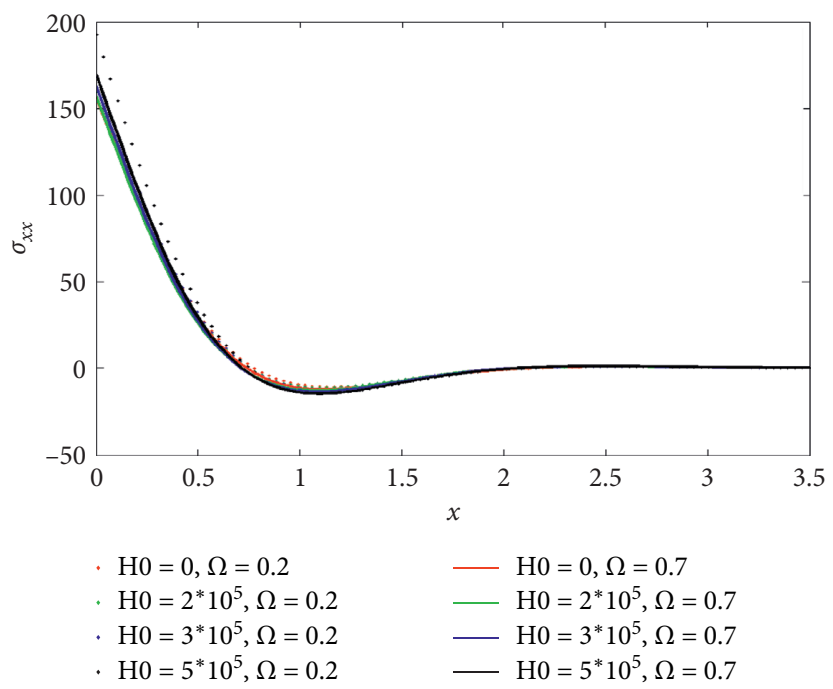

FIgURE 28: Stress $\sigma_{x x}$ concerning $x$ with magnetic and rotation. 


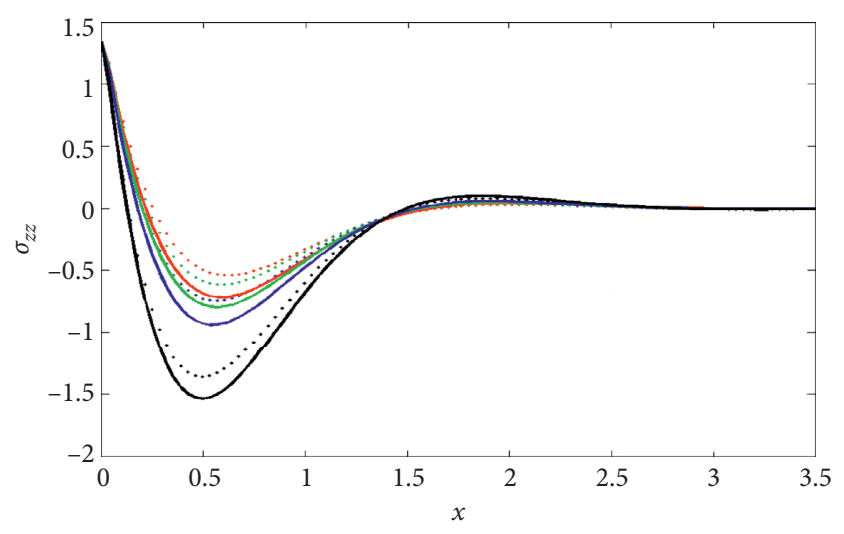

$$
\begin{aligned}
& \mathrm{H} 0=0, \Omega=0.2 \\
& \text { H } 0=2^{*} 10^{5}, \Omega=0.2 \\
& \text { H0 }=3^{*} 10^{5}, \Omega=0.2 \\
& \text { H0 }=5^{*} 10^{5}, \Omega=0.2
\end{aligned}
$$$$
-\mathrm{H} 0=0, \Omega=0.7
$$$$
\mathrm{H} 0=2^{*} 10^{5}, \Omega=0.7
$$$$
-\mathrm{H} 0=3^{*} 10^{5}, \Omega=0.7
$$$$
-\mathrm{H} 0=5^{*} 10^{5}, \Omega=0.7
$$

Figure 29: Stress $\sigma_{z z}$ concerning $x$ with magnetic and rotation.

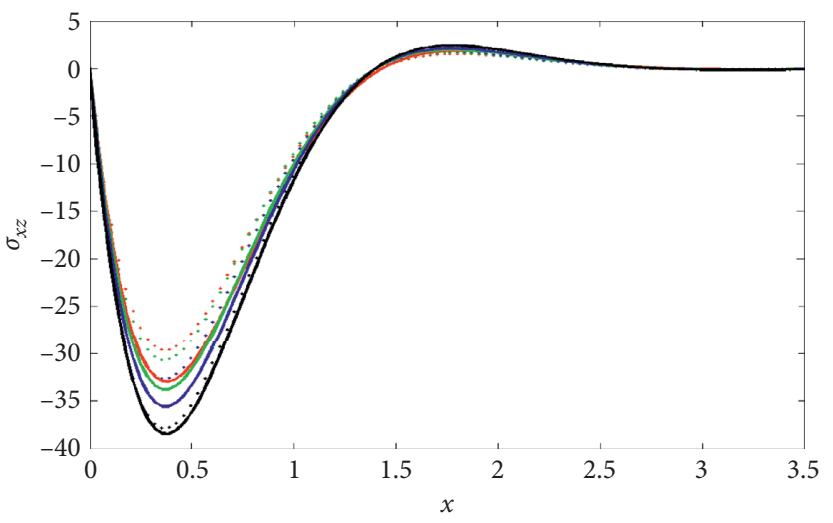

$$
\begin{array}{ll}
\mathrm{H} 0=0, \Omega=0.2 & -\mathrm{H} 0=0, \Omega=0.7 \\
\mathrm{H} 0=2^{*} 10^{5}, \Omega=0.2 & -\mathrm{H} 0=2^{*} 10^{5}, \Omega=0.7 \\
\mathrm{H} 0=3^{*} 10^{5}, \Omega=0.2 & -\mathrm{H} 0=3^{*} 10^{5}, \Omega=0.7 \\
\mathrm{H} 0=5^{*} 10^{5}, \Omega=0.2 & -\mathrm{H} 0=5^{*} 10^{5}, \Omega=0.7
\end{array}
$$

FIGURE 30: Stress $\sigma_{x z}$ concerning $x$ with magnetic and rotation.

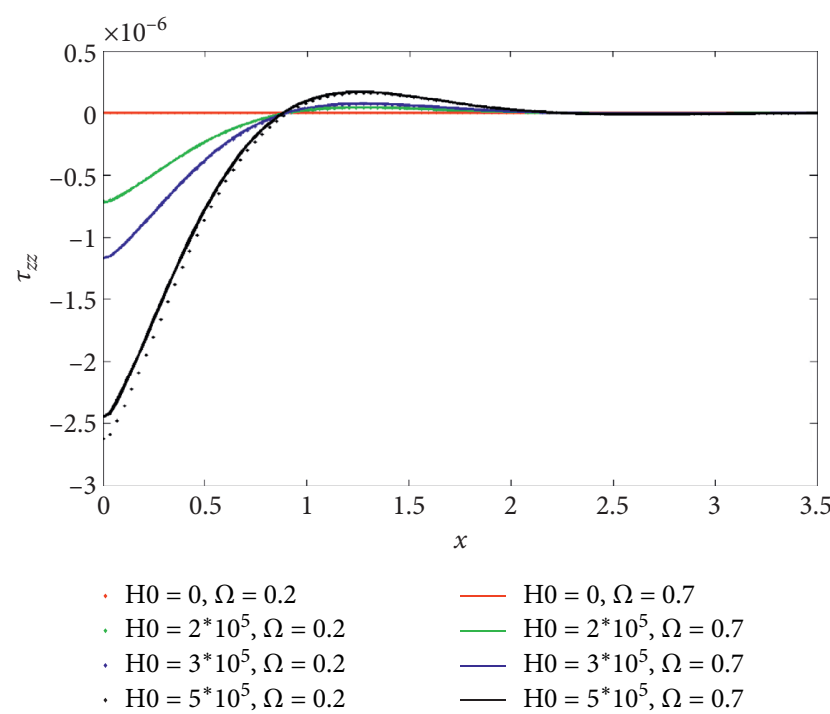

Figure 31: Stress $\tau_{z z}$ concerning $x$ with magnetic and rotation.

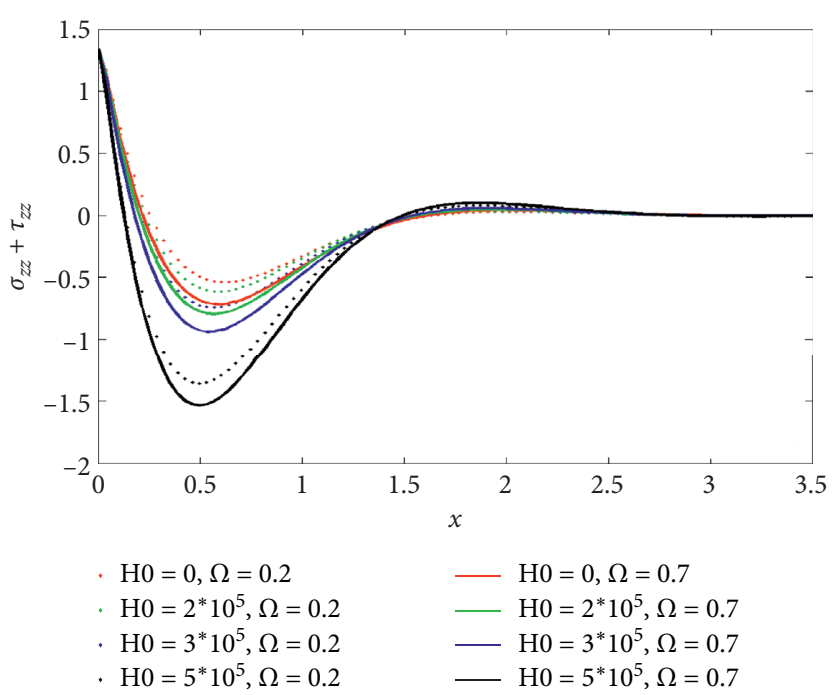

Figure 32: Stress $\tau_{z z}+\sigma_{z z}$ concerning $x$ with magnetic and rotation.

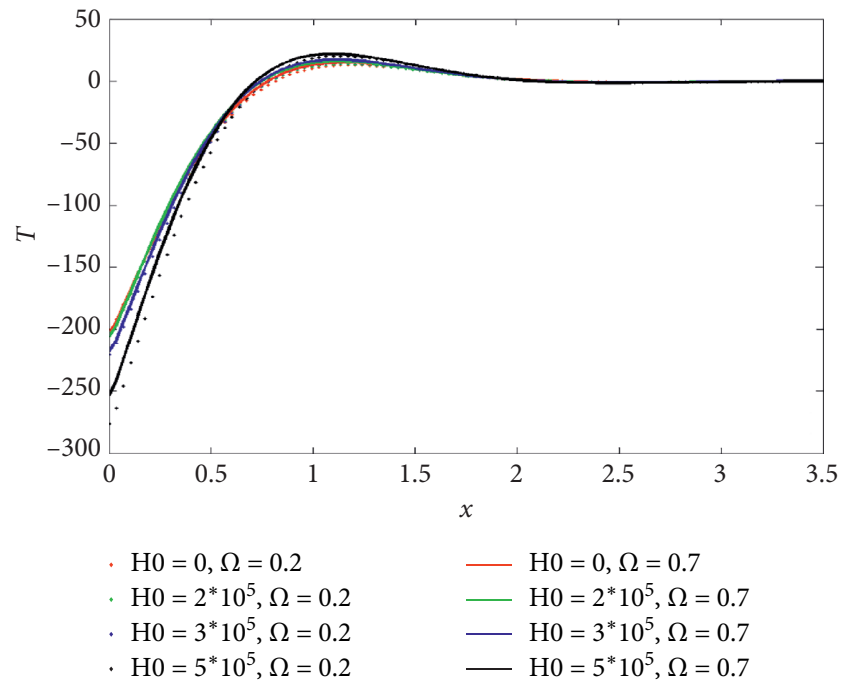

Figure 33: Temperature $T$ concerning $x$ with magnetic and rotation.

$\tau_{0}=1, \vartheta_{0}=0$. We show that the displacement, stresses, and temperature demonstrates clearly the difference between the coupled and the generalized theories of thermoelasticity (see Figures 2-9). It implies that applying G-L theory provides good results compared with the remaining theories, and this physically indicates the developed generalized thermoelastic theories in the positive direction. Figures 10-17 display the values of the displacement, stresses, and temperature with respect to $x$-axis for different values of gravity field $g=0,5,7,9.8$ considering two values of rotation $\Omega=0.2, \ldots, \Omega=0.7_{--.}$. It appears that $u, \sigma_{z z}, \sigma_{x z}, \tau_{z z}, \sigma_{z z}+$ $\tau_{z z}$, and $T$ decreased (i.e., affects negatively) with the increased values of the rotation but increased (i.e., affects positively) concerning the remaining distributions, which indicates strong effect with the strong values of rotation. It is observed that the component of displacement $u$ decreases in the interval $0<x<1.3$ with an increasing gravity field but increases in the 
interval $[1.3,2.5]$, while it coincides if $x>2.5$ tends to zero, as well as the displacement component $w$ decreases with increasing gravity field in the interval $[0,0.8]$, but increases in the interval $0.8<x<1.8$ and it coincides if $x>1.8$. From Figure 12, it obvious that the stresses $\sigma_{x x}, \sigma_{z z}, \tau_{z z}, \sigma_{z z}+\tau_{z z}$ and temperature $T$ increase, decrease, and then coincide tending to zero as distance tends to infinity that agrees with the physical meaning of the phenomena (i.e., if the wave is far from the origin, then the distributions tend to infinity "unknown"), but the shear stress $\sigma_{x z}$ decreases with an increasing gravity field. The distributions are shown graphically in Figures 18-25 considering variation in laser pulse: $t=0,0.3$, $0.6,0.9$, and it obvious that the displacement components $u$ and $w$, stresses $\sigma_{z z}, \tau_{z z}, \sigma_{z z}+\tau_{z z}$, and temperature $T$ increase, decrease, and then coincide tending to zero as distance $x$ tends to infinity, but only the normal stress $\sigma_{x x}$ decreases with an increasing gravity. Figures $26-33$ plot the variation in the displacement and stresses components, also the temperature distribution with respect to the distance and various values of the magnetic field and two values of rotation. It shows that the magnetic field has a strong sense on all the distributions; we concluded that, except the normal stress on the $x$-direction which makes a slight decreasing change with an increasing $\mathrm{H} 0$, one can be obvious that $u, w, \sigma_{z z}, \tau_{z z}, \sigma_{z z}+\tau_{z z}$, and $T$ increased with an increasing magnetic field.

Physically, the gravity, magnetic field, laser pulse, and thermoelasticity with the model theories have a significant effect on the propagation of the wave phenomenon.

Finally, it strong obvious that all variables calculated satisfied the boundary conditions exactly either the calculus or the graphs (the figures display total normal stress $\sigma_{z z}+\tau_{z z}$, shear stress $\sigma_{x z}$, and temperature distribution $T$ ).

If the gravity and magnetic field considered the thermal loading influence due to laser pulse on generalized micropolar-thermoelastic solid with comparison of different theories, the results obtained have been deduced and are in agreement with the previous results by Othman et al. [7].

On the contrary, when the laser pulse and gravity are neglected, the obtained results are deduced as a special case from the present investigation and are in agreement with the results obtained by Abo-Dahab et al. [15].

\section{Conclusion}

In the view of illustrating the numerical results, we concluded the following remarks:

(1) The technique of normal mode technique is used in wide range of applications in diverse fields such as engineering, geophysics, thermodynamics, geology, acoustics, eyes medicine, and engineering.

(2) The magnetic field, rotation, gravity, and laser pulse have a significant role in temperature, displacements, and stresses and all the physical quantities by decreasing or increasing.

(3) The results described for the medium of crystal, which may provide interesting information for the experimental researchers work on this field, satisfy the boundary conditions.
(4) The values of all physical quantities converge to zero with an increasing distance $x$, and all functions are exactly continuous.

(5) Applying G-L theory has good results compared with the remaining theories, and this physically indicates the developed thermoelastic theories in the positive direction.

(6) The results obtained should be useful for researchers/scientists in designing new materials, material sciences, physicists, and the development of the magneto-thermoelasticity and in practical situations such as in optics, geophysics, petroleum extraction, acoustics, and oil prospecting.

Finally, we conclude that the results obtained have a significant rule in engineering, astronomy, aircrafts, dynamical system reactors, and aircrafts.

\section{Nomenclature}

\begin{tabular}{|c|c|}
\hline A: & Wave number \\
\hline B: & Induced magnetic field vector \\
\hline$C_{e}:$ & Specific heat at constant strain \\
\hline E: & Induced electric field vector \\
\hline$e_{i j}$ : & Strain tensor components \\
\hline$F_{i}:$ & Lorentz force tensor \\
\hline$f(t):$ & Temporal profile \\
\hline$g:$ & Acceleration due to gravity \\
\hline$G_{i}$ : & Gravity force tensor \\
\hline H: & Initial uniform magnetic intensity vector \\
\hline$I_{0}$ : & Absorbed energy \\
\hline J: & Current density vector \\
\hline K: & Thermal conductivity \\
\hline$k^{*}:$ & Characteristic of the GN II theory constant \\
\hline$n_{0}, n_{1}:$ & Parameters \\
\hline$p_{1}:$ & Mechanical force magnitude \\
\hline$Q:$ & Heat input of the laser pulse \\
\hline$r:$ & Beam radius \\
\hline$T:$ & Absolute temperature \\
\hline$T_{0}:$ & $\begin{array}{l}\text { Reference temperature of the medium } \\
\left|\left(T-T_{0}\right) / T_{0}\right| \leq 1\end{array}$ \\
\hline$t:$ & Time variable \\
\hline$t_{0}:$ & Pulse rising time \\
\hline$u, v, w:$ & Displacement components \\
\hline$x, y, z:$ & Coordinates of the system \\
\hline$\nabla^{2}:$ & Laplacian operator \\
\hline$\alpha_{t}:$ & Thermal expansion \\
\hline$\delta_{i j}:$ & Kronecker delta \\
\hline$\varepsilon_{1}, \varepsilon_{2}, \varepsilon_{3}$ & Coupling constants \\
\hline$\gamma=\alpha_{t}(3 \lambda+2 \mu)$ & Material constant \\
\hline$\lambda, \mu:$ & Lame's constants \\
\hline$\rho:$ & Density \\
\hline$\vartheta_{0}, \tau_{0}:$ & Thermal relaxation times \\
\hline$\sigma_{i j}:$ & Stress tensor components \\
\hline$\mu_{e}:$ & Magnetic permeability \\
\hline$\omega:$ & Angular frequency \\
\hline$\Omega:$ & Angular velocity. \\
\hline
\end{tabular}




\section{Data Availability}

The data used to support the findings of this study are available from the corresponding author on request.

\section{Conflicts of Interest}

The authors declare that they have no conflicts of interest.

\section{Acknowledgments}

This research was funded by the Deanship of Scientific Research, Taif University, KSA (research group number 11441-105).

\section{References}

[1] M. A. Biot, "Thermoelasticity and irreversible thermodynamics," Journal of Applied Physics, vol. 27, no. 3, pp. 240-253, 1956.

[2] H. W. Lord and Y. Shulman, "A generalized dynamical theory of thermoelasticity," Journal of the Mechanics and Physics of Solids, vol. 15, no. 5, pp. 299-309, 1967.

[3] A. E. Green and K. A. Lindsay, "Thermoelasticity," Journal of Elasticity, vol. 2, no. 1, pp. 1-7, 1972.

[4] A. E. Green and P. M. Naghdi, "Thermoelasticity without energy dissipation," Journal of Elasticity, vol. 31, no. 3, pp. 189-208, 1993.

[5] A. E. Green and P. M. Naghdi, "On undamped heat waves in an elastic solid," Journal of Thermal Stresses, vol. 15, no. 2, pp. 253-264, 1992.

[6] T. J. I. A. Bromwich, "On the influence of gravity on elastic waves, and, in particular on the vibrations of an elastic globe," in Proceedings of the London Mathematical Society, vol. 30, no. 1 , pp. 98-165, 1898.

[7] M. I. A. Othman, W. M. Hasona, and E. E. M. Eraki, "Influence of gravity field and rotation on a generalized thermoelastic medium using a dual-phase-lag model," Journal of Thermoelasticity, vol. 1, pp. 12-22, 2013.

[8] S. C. Das, D. P. Acharya, and P. R. Sengupta, "Surface waves in an inhomogeneous elastic medium under the influence of gravity," Revue Roumaine des Sciences Techniques.vol. 37, pp. 539-551, 1992.

[9] A. M. Abd-Alla, S. M. Abo-Dahab, and H. A. Alotaibi, "Propagation of a thermoelastic wave in a half-space of a homogeneous isotropic material subjected to the effect of gravity field," Archives of Civil and Mechanical Engineering, vol. 17, no. 3, pp. 564-573, 2017.

[10] A. M. Abd-Alla, S. M. Abo-Dahab, and A. Khan, "Rotational effect on thermoelastic Stoneley, Love and Rayleigh waves in fibre-reinforced anisotropic general viscoelastic media of higher order," Structural Engineering and Mechanics, vol. 61, no. 2, pp. 221-230, 2017.

[11] S. M. Abo-Dahab, A. M. Abd-Alla, and H. A. Alotabi, "On influence of thermal stress and magnetic field in thermoelastic half-space without energy dissipation," Journal of Thermal Stresses, vol. 40, pp. 213-230, 2017.

[12] S. M. Abo-Dahab, A. M. Abd-Alla, and I. H. EL-Sirafy, "Effect of gravity field, initial stress and rotation on the S-waves propagation in a non-homogeneous anisotropic medium with magnetic field," Journal of Mechanical Science and Technology, vol. 28, no. 8, pp. 3003-3011, 2014.

[13] S. M. Abo-Dahab and K. Lotfy, "Two-temperature plane strain problem in a semiconducting medium under photothermal theory," Waves in Random and Complex Media, vol. 27, no. 1, pp. 67-91, 2017.

[14] S. M. Abo-Dahab, M. I. A. Othman, and O. N. S. Alsebaey, "Effect of two temperature and rotation on plane waves of generalized thermoelasticity under thermal loading due to laser pulse in the context of three theories," Journal of Scientific and Engineering Research, vol. 3, no. 6, pp. 173-187, 2016.

[15] S. M. Abo-Dahab, A. M. Abd-Alla, and A. J. Alqarni, "A twodimensional problem with rotation and magnetic field in the context of four thermoelastic theories," Results in Physics, vol. 7, pp. 2742-2751, 2017.

[16] K. Lotfy and M. E. Gabr, "Response of a semiconducting infinite medium under two temperature theory with photothermal excitation due to laser pulses," Optics \& Laser Technology, vol. 97, pp. 198-208, 2017.

[17] S. M. Abo-Dahab, "Reflection of generalized magneto-thermoelastic waves with two temperatures under influence of thermal shock and initial stress," Journal of Heat Transfer, vol. 140, no. 10, p. 8, Article ID 102005, 2018.

[18] P. K. Saroj, S. A. Sahu, A. Singhal, and S. M. Abo-Dahab, "On the transference of love-type waves in pre-stressed PZT-5H material stick on $\mathrm{SiO} 2$ material with irregularity," Materials Research Express, vol. 6, no. 12, Article ID 125703, 2019.

[19] S. M. Abo-Dahab and S. Biswas, "P, T, and SV wave propagation at the interface between solid-liquid media with magnetic field and initial stress in the context of three-phase-lag model," Mechanics of Advanced Materials and Structures, vol. 27, no. 2, pp. 165-175, 2020.

[20] A. M. Abd-Alla, S. M. Abo-Dahab, S. M. Ahmed, and M. M. Rashid, "Rayleigh surface wave propagation in an orthotropic rotating magneto-thermoelastic medium subjected to gravity and initial stress," Mechanics of Advanced Materials and Structures, vol. 27, no. 16, pp. 1400-1411, 2020.

[21] S. M. Abo-Dahab, A. M. Abd-Alla, and A. Alqarni, "Magnetothermoelastic problem in the context of four theories under influence of Laser pulse and gravity field," Archives of Thermodynamics, vol. 41, no. 1, pp. 31-66, 2020.

[22] S. Marzougui, M. Bouabid, F. Mebarek-Oudina, N. AbuHamdeh, M. Magherbi, and K. Ramesh, "A computational analysis of heat transport irreversibility phenomenon in a magnetized porous channel," International Journal of $\mathrm{Nu}$ merical Methods for Heat \& Fluid Flow, 2020.

[23] S. M. Dahab, M. A. Abdelhafez, F. Mebarek-Oudina, and S. M. Bilal, "MHD casson Nanofluid flow over non-linearly heated porous medium in presence of extending surface effect with suction/injection," Indian Journal of Physics, 2020.

[24] M. I. A. Othman, W. M. Hasona, and E. M. Abd-Elaziz, "The influence of thermal loading due to laser pulse on generalized micropolarthermoelastic solid with comparison of different theories," Multidiscip. Model. Mater. Struct, vol. 10, pp. 328-345, 2014.

[25] R. S. Dhaliwal and A. Singh, Dynamic Coupled Thermoelasticity, Hindustan Publishing Corporation, New Delhi, India, 1980. 